\title{
3 Méthode
}

Avant de présenter les trois méthodes utilisées dans le cadre de la présente étude (cf. 3.3 : questionnaires sur les représentations et attitudes ; 3.4 : analyse de voyelles produites par des locuteurs-modèles ; 3.5 : évaluation des voyelles des locuteurs-modèles par des auditeurs «non experts »), il est tout d'abord nécessaire, d'une part, de différencier les trois perspectives combinées dans cette étude (productions, perceptions, représentations/attitudes ; cf. 3.1) et, d'autre part, d'évoquer les principes fondamentaux de la recherche empirique en sciences sociales sur lesquels repose le choix des méthodes (cf. 3.2).

\subsection{Productions, perceptions, représentations et attitudes}

La distinction conceptuelle entre les productions, les attitudes, les représentations et les perceptions peut être expliquée sur la base de l'utilisation qu'en fait la 'linguistique perceptive des variétés' (all. perzeptive Varietätenlinguistik ; cf. Pustka 2007 ; 2008 ; Krefeld/Pustka 2010 ; Postlep 2010). Remontant à Krefeld $(2005,162)$, ce cadre théorique étudie la relation entre 'conscience linguistique' (all. Sprachwissen) et 'parole' (all. Sprechtätigkeit). La parole correspond aux productions se situant dans l'usage concret du langage. La conscience linguistique regroupe, pour sa part, les représentations et attitudes - qui peuvent être basées ou non sur des perceptions concrètes de productions linguistiques.

Les représentations peuvent toucher, d'une part, aux structures de la langue au sens strict du terme, c'est-à-dire à la capacité de parler et de comprendre une langue (cf. Chomsky ${ }^{2} 2000,83$ ). D'autre part, elles peuvent porter sur la structuration cognitive de la variation linguistique (cf. Postlep 2010, 55). Les attitudes, qui remontent à la 'linguistique populaire' (folk linguistics, cf. Preston/Niedzielski 2000) et se basent sur la recherche en psychologie sociale (cf. Werlen 1984), sont pour leur part définies comme étant les évaluations et sentiments affectifs face à la variation linguistique (cf. Postlep 2010, 55-56). Au sein de la linguistique, elles permettent en particulier d'étudier le statut (plus ou moins prestigieux ou stigmatisé) de langues et de variétés (cf. Glück 2000, 178 ; Krefeld/Pustka 2010, 15).

Aussi bien les représentations que les attitudes linguistiques peuvent être générées non seulement par la perception de productions linguistiques concrètes, mais aussi par des facteurs extralinguistiques (p. ex. l'appartenance du locuteur à une catégorie socio-économique et/ou ethnique particulière, cf. Didelot 2019, 119). Les représentations et attitudes peuvent donc être perceptives ou non. Or, c'est aux représentations ou attitudes perceptives que se réfère le terme de perceptions utilisé 
systématiquement dans le présent ouvrage : il s'agit des représentations ou attitudes systématiquement liées à la perception de productions linguistiques concrètes dans une situation de communication réelle (cf. Krefeld/Pustka 2010, 14).

Il est cependant important de mentionner que les représentations et attitudes perceptives et non perceptives s'influencent mutuellement : d'une part, les représentations et attitudes se basent sur des perceptions et sont constamment modifiées par ces dernières ; d'autre part, les perceptions sont souvent filtrées par les représentations non perceptives existantes. Ainsi, des attitudes touchant à la prononciation pourront être fortement influencées par les représentations géographiques, sociales ou situationnelles que les auditeurs associent à la variété entendue (cf. Didelot 2019, 119 ; cf. également Lippi-Green 2012 ; Moyer 2013). En France septentrionale, par exemple, un écart linguistique par rapport à la norme sera souvent perçu comme étant de nature géographique même s'il est plutôt spécifique à un groupe social défavorisé (cf. Hauchecorne/Ball 1997, 22).

\subsection{Principes fondamentaux de la recherche empirique en sciences sociales}

En ce qui concerne les principes fondamentaux de la recherche empirique en sciences sociales, mentionnons tout d'abord les trois critères fondamentaux de qualité de telles recherches empiriques : l'objectivité, la fiabilité et la validité (cf. Adams 1936, 329-335 ; König 2010, 495-498, Diekmann ${ }^{6} 2012$, 479-488). Rappelons que l'objectivité de questions ou de mesures est garantie lorsque les réponses obtenues ou les valeurs mesurées sont indépendantes de l'enquêteur (cf. König 2010, 495). La fiabilité fait référence au fait que ces réponses et valeurs mesurées ne doivent pas être soumises aux hasards circonstanciels, de sorte qu'une étude effectuée postérieurement devrait engendrer des résultats ou valeurs stables par rapport à la précédente (cf. König 2010, 495-496). Finalement, la validité d'une question est garantie lorsqu'elle est en adéquation avec ses objectifs, c'est-à-dire lorsqu'elle mesure effectivement ce qu'elle est censée mesurer (cf. König 2010, 497-498). Notons que les méthodes non structurées, comme l'observation et l'entretien non directif (des méthodes considérées comme principalement qualitatives) auront plutôt tendance à être valides, mais moins objectives et fiables. Les méthodes structurées, comme le questionnaire ou l'entretien directif (des méthodes considérées comme principalement quantitatives), auront plutôt tendance à être plus objectives et fiables, mais moins valides. Finalement, certaines méthodes semi-structurées, comme l'entretien semi-directif, se trouvent entre ces deux pôles. 
Mentionnons par ailleurs un autre objectif que toute étude se voulant généralisable aux sous-groupes étudiés devrait viser : l'obtention de résultats s'approchant d'une certaine représentativité (cf. Leech 1991, 9 ; Manning/Schütze 1999, 119 ; Leech 2006, 135). Cependant, comme le souligne Sinclair (2004), il est évident qu'aucun sondage ne peut prétendre être totalement représentatif :

« Everyone seems to accept that no limits can be placed on a natural language [. . .]. Therefore no corpus, no matter how large, how carefully designed, can have exactly the same characteristics as the language itself. [.. .] So we sample, like all the other scholars who study unlimitable phenomena. We remain, as they do, aware that the corpus may not capture all the patterns of the language, nor represent them in precisely the correct proportions " (Sinclair 2004, 2).

C'est donc bien l'échantillonnage, c'est-à-dire la méthode de sélection des informateurs qui formeront la base de données de l'ouvrage, qui donne accès à cette représentativité (cf. Arbach/Ali 2013, 2). Cette question est abordée ci-dessous pour chacune des trois méthodes utilisées dans le présent ouvrage (cf. 3.3.2, 3.4.1.1, 3.5.4).

Ces différents principes généraux se trouvent donc à la base des choix de méthodes de toute étude empirique en sciences sociales. Pour ce qui est du présent ouvrage en particulier, un rappel de ses deux principaux objectifs aidera à en comprendre le choix des méthodes :

1. D’une part, l'ouvrage a pour objectif de mettre en lumière une éventuelle volonté ressentie par les informateurs « non experts » parisiens, suisses romands et québécois de codifier des normes de prononciation propres à chacune de ces régions.

2. D’autre part, il s'agira de définir, à l'exemple des voyelles, les traits de prononciation appartenant à ces normes potentielles.

Le premier des deux objectifs portant sur l'aspect des représentations et attitudes (cf. Krefeld/Pustka 2010, 12), le choix de la méthode a dû se faire entre les deux méthodes les plus courantes dans le domaine de la sociolinguistique : l'entretien et le questionnaire, qui présentent tous deux des avantages et des inconvénients. Si l'entretien présente « l'avantage de placer l'enquêteur et l'enquêté dans une relation humaine, souple et coopérative » (Boukous 1999, 24), cette méthode engendre en général également une réduction de la taille de l'échantillon pris en compte, étant donnés le temps nécessaire ainsi que les moyens matériels et financiers requis. Par ailleurs, une telle méthode est moins maîtrisable car plus sensible à la subjectivité, la situation de l'entretien (endroit de l'entretien, personnalité, humeur, apparence, usage linguistique de l'enquêteur, etc.) pouvant influencer les résultats de manière incontrôlée (cf. Boukous 1999, 24). 
Le principal avantage du questionnaire, méthode structurée sur support écrit, est qu'il se prête à une analyse quantitative (cf. Boukous 1999, 15). Il permet en effet de travailler avec un échantillon plus large et de manière systématique, et ce, en peu de temps et avec peu de ressources. Par ailleurs, les réponses obtenues sont souvent plus sincères car l'anonymat est mieux garanti sous forme écrite. Cependant, les questions d'un tel questionnaire doivent être claires, bien ordonnées et dénuées d'ambiguïté pour tous les informateurs, faute de quoi certaines restent sans réponse ou engendrent des types de réponse qui n'étaient pas visés par la question. Par ailleurs, le temps nécessaire au questionnaire ne doit pas être trop long pour éviter que de nombreux informateurs n'interrompent l'enquête prématurément (cf. Boukous 1999, 24).

Le premier objectif, qui touche aux représentations et attitudes (cf. 3.1), visant une certaine représentativité des trois populations étudiées (qui n'est atteignable qu'à travers un échantillon assez large) et des réponses comparables (favorisées par une certaine systématicité de l'enquête), la méthode du questionnaire sur support écrit a été préférée à celle de l'entretien.

Le deuxième objectif du présent ouvrage, la définition des voyelles appartenant aux trois potentielles normes de prononciation (cf. supra), n'est, pour sa part, pas lié aux représentations et attitudes globales des informateurs par rapport à la norme de prononciation, mais aux domaines des productions et des perceptions (cf. 3.1) : d'une part, il est en effet nécessaire de procéder à une analyse acoustique des voyelles produites par les locuteurs du groupe considéré comme le modèle à suivre par les « non-experts » (les locuteurs-modèles, cf. 2.2.1.3) pour pouvoir émettre des hypothèses concrètes quant aux voyelles appartenant aux normes des trois régions étudiées. D’autre part, dans le sens de la définition d'une norme démocratique (cf. 2.2.1.4), ces hypothèses devront être confirmées par des tests de ces traits de prononciation dans la perception d'un échantillon le plus représentatif possible de locuteurs « non experts » de chacune des régions étudiées.

Ainsi, c'est sur la base de cette combinaison d'un sondage global sur les représentations et attitudes, d'une analyse acoustique des voyelles produites par des locuteurs-modèles et de tests perceptifs de ces mêmes voyelles qu'une définition relativement fiable et généralisable de ces normes de prononciation peut être proposée. C'est donc sur la combinaison de ces trois méthodes que se fonde le présent ouvrage, trois méthodes qui seront présentées dans ce chapitre :

- Le premier sous-chapitre portera sur les questionnaires écrits touchant aux représentations et attitudes de locuteurs « non experts » face à différents aspects liés à la norme (de prononciation) (cf. 3.3).

- Le deuxième sous-chapitre touchera aux analyses acoustiques de voyelles produites par des locuteurs-modèles qui ont permis la mise en place d'hypo- 
thèses quant aux traits de prononciation appartenant potentiellement à ces normes (cf. 3.4).

- Le dernier sous-chapitre abordera le test de ces hypothèses dans le cadre d'expériences de perception : les informateurs « non experts » provenant de ces trois régions ont dû y évaluer l'utilisation de ces traits selon différents types de questions (touchant à différentes situations de communication) et de réponses (échelles et réponses à choix simple) (cf. 3.5).

\subsection{Représentations et attitudes : questionnaires}

La première méthode utilisée dans ce travail concerne les représentations et attitudes. La méthode se présente sous la forme de questionnaires principalement quantitatifs (avec quelques ajouts qualitatifs) distribués à Paris, en Suisse romande et au Québec selon un échantillonnage par quotas. Ils touchent aux représentations et attitudes d'informateurs «non experts » face au processus de création d'une norme propre à chacune de ces régions. Le questionnaire aborde ainsi aussi bien les auto-représentations des Parisiens/Suisses romands/Québécois face à leur propre prononciation que leurs hétéro-représentations de la norme de prononciation traditionnelle et des autres prononciations francophones. La présentation de cette méthode sera divisée en deux parties : je présenterai d'abord les différentes questions du questionnaire et montrerai leur pertinence pour les objectifs de l'étude (cf. 3.3.1), avant d'aborder la question de la sélection et du recrutement des informateurs (cf. 3.3.2).

\subsubsection{Questionnaires}

Le questionnaire contient 17 questions groupées en cinq sections thématiques ayant pour objectif de couvrir différents aspects liés à la norme (de prononciation) (cf. annexe 1 : Paris ; annexe 2 : Suisse romande ; annexe 3 : Québec) : les auto- et hétéro-représentations, la valorisation et la dépréciation de prononciations endogènes et exogènes, le prestige latent et manifeste de ces mêmes prononciations et l'insécurité linguistique y étant liée (cf. 2.1.2). Les cinq sections, suivies d'une sixième portant sur les données sociodémographiques récoltées à la fin du questionnaire, se structurent de la manière suivante selon l'aperçu proposé dans le tableau 10.

Trois précisions sont encore nécessaires quant à la présentation des trois questionnaires ci-dessous : premièrement, je tiens à souligner que dans l'élaboration des questions, j'ai pris en compte le statut sociolinguistique différent de 
Tableau 10 : Structure du sous-chapitre sur les questionnaires.

\begin{tabular}{ll}
\hline Sous-chapitres & Sections \\
\hline 3.3.1.1 & Représentations des accents de la francophonie \\
\hline 3.3.1.2 & Représentations des accents des régions étudiées \\
\hline 3.3 .1 .3 & Représentations des différences d'accent à l'intérieur ces régions \\
\hline 3.3 .1 .4 & $\begin{array}{l}\text { Représentations de la prononciation des présentateurs de télévision de ces } \\
\text { régions }\end{array}$ \\
\hline 3.3.1.5 & $\begin{array}{l}\text { Attitudes face à la prononciation de chaque région dans une comparaison } \\
\text { avec les prononciations d'autres régions francophones }\end{array}$ \\
\hline 3.3.1.6 & Informations sociodémographiques \\
\hline
\end{tabular}

la Suisse romande et du Québec (considérés comme " périphériques »; cf. p. ex. Lafontaine 1986 ; Singy 1996) par rapport à celui de Paris (considéré comme « central », cf. Valdman 1982 ; Gagné 1983). C’est la raison pour laquelle le questionnaire parisien contient certaines asymétries par rapport aux questionnaires suisses romands et québécois : certaines questions n'étaient en effet aptes à être posées que dans les «périphéries » (questions 4, 11, 12, 13 ; cf. 3.3.1.1), d’autres ont dû être posées selon des perspectives contraires au centre et en périphérie (questions 8, 9, 10, 15, 16 ; cf. 3.3.1.1). Deuxièmement, en raison de ces asymétries, la numérotation des questions présentée ci-dessous ne correspond pas dans tous les cas à celle qui pourra être trouvée dans les questionnaires originaux (cf. annexes 1-3). Troisièmement, il faut noter que dans la présentation des questions, pour des raisons de clarté et d'économie de place, les questions identiques des questionnaires parisiens, suisses romands et québécois ont été réunies autant que possible dans un seul énoncé en utilisant des barres obliques pour séparer les régions les unes des autres.

\subsubsection{Section 1 : représentations des accents de la francophonie}

Question 1: Quels accents de français connaissez-vous dans le monde?

La première question (cf. question 1) est une question d'introduction, nommée également « question brise-glace » (all. Eisbrecherfrage ; cf. Diekmann ${ }^{6} 2012$, 483-485). Elle favorise l'entrée en matière des informateurs, les met en confiance et, dans l'idéal, suscite leur intérêt pour les questions de prononciation. Elle a donc été formulée de façon ouverte et générale et ne touche pas directement aux questions de normes de prononciation, mais aux questions plus générales d'ac- 
cents du français dans le monde. Notons également qu'il s'agit d'une question ouverte pour laquelle aucune possibilité de réponse préétablie n’a été donnée aux informateurs.

Question 2 : Selon vous, où a-t-on le moins d'accent dans le monde?

La deuxième question (cf. question 2) aborde, pour sa part, l'un des principaux aspects de la norme de prononciation dans la conscience des informateurs : les représentations de la variété considérée comme norme comme étant (perceptivement) « neutre ». Dans cette première section, cette question touche à la francophonie dans sa globalité. Il s'agit par ailleurs, ici aussi, d'une question ouverte sans choix de réponses fixé au préalable, la quantification des réponses ayant dû être faite à posteriori.

\subsubsection{Section 2 : représentations des accents des régions étudiées}

Question 3.1: Les Parisiens/Suisses romands/Québécois ont-ils un accent ? ( $\square$ Oui $\square$ Non) Questions 3.2 : Si oui, à quoi peut-on les reconnaitre le plus? ( $\square$ À leur vocabulaire, $\square$ À leur prononciation, $\square$ À leur grammaire, $\square$ À autre chose)

La deuxième section porte sur les représentations des différences perçues à l'intérieur de chaque région. Elle commence par une question qui visait, d'une part, à tester les auto-représentations des informateurs de chaque région par rapport à la « neutralité » perçue de leur prononciation à l'intérieur de leur région (cf. question 3.1). D’autre part, la question avait pour objectif de découvrir à quel niveau linguistique (vocabulaire, prononciation, grammaire) ces mêmes informateurs pensent percevoir le plus grand nombre de différences entre l'accent de leur région et les autres accents. C'est la raison pour laquelle la question a été complétée par une question touchant à ces niveaux linguistiques (cf. question 3.2). Les informateurs pouvaient y répondre sur la base d'un choix simple parmi quatre possibilités de réponse (à leur vocabulaire, à leur prononciation, à leur grammaire, à autre chose) qu'ils pouvaient préciser à l'aide d'exemples formulés librement. La combinaison des réponses à choix simple avec des exemples formulés par les informateurs présente en effet l'avantage de permettre, d'une part, la quantification des résultats et, d'autre part, leur vérification de manière qualitative. Les informateurs auront en effet souvent tendance à nommer plus de traits (des schibboleths ; cf. Glück 2000, 603 ; Krefeld/Pustka 2010, 20) aux niveaux linguistiques (prononciation, lexique, grammaire) les plus saillants dans leur conscience. Notons que ce type de combinaison de questions fermées et ouvertes 
s'est déjà avéré fructueux dans plusieurs enquêtes sociolinguistiques préalables (cf. Boukous 1999, 17-18 ; des exemples de ce type de combinaison pourront être trouvés chez Reutner 2005 ; Pustka 2010).

Question 4.1 : Y a-t-il des Suisses romands / Québécois qui essaient de perdre leur accent? ( $\square$ Oui $\square$ Non)

Question 4.2 : Pourquoi (pas)?

La deuxième question de cette section (cf. question 4.1/4.2) touche non plus aux représentations, mais aux attitudes des informateurs face à leur propre prononciation : il s'agit ici de mettre en lumière la potentielle insécurité linguistique statutaire (cf. Remysen 2004, 97) qu'ils pourraient ressentir en considérant leur propre prononciation comme moins prestigieuse que celle du modèle de référence. Étant donné que la question touche au phénomène de l'insécurité linguistique des locuteurs « périphériques » face aux locuteurs parisiens, la question n’a pas été posée dans le questionnaire parisien. Afin d'éviter autant que possible tout risque de biais de réponse en termes de désirabilité sociale (cf. entre autres Paulhus 2002, 49-69), la question a été formulée non pas du point de vue personnel des informateurs, mais dans la perspective d'un observateur externe (cf. Avetisyan 2012, 3). Les informateurs pouvaient y répondre par oui ou non et justifier librement leur réponse (Pourquoi (pas) ?). Notons que la question est inspirée d'une question similaire posée par Singy (1996, 286, question 23) ${ }^{1}$ à des informateurs du canton de Vaud, en Suisse.

\subsubsection{Section 3 : représentations des différences d'accent à l'intérieur des régions étudiées}

Question 5 : Selon vous, où a-t-on le moins d'accent en France/en Suisse romande/au Québec?

Cette section aborde les différences de prononciation perçues à l'intérieur de chacune des régions. Elle est constituée d'une question (cf. question 5) similaire à la question 2 (cf. supra), à la différence qu'elle ne touche pas à la francophonie dans sa globalité, mais uniquement aux accents se trouvant dans chacune des régions étudiées. La question concerne donc les représentations de la variété considérée à l'intérieure de chacune des régions comme norme (perceptivement)

1 Question 23 du questionnaire de Singy $(1996,286)$ : «Y a-t-il des Vaudois qui essaient de perdre leur accent ? »; possibilités de réponse : « oui » ou « non ». 
« neutre ». Par ailleurs, il s'agit, ici aussi, d'une question ouverte sans possibilités de réponse préétablies.

Notons par ailleurs que dans le questionnaire, cette question est précédée d'une question supplémentaire utilisant la méthode des cartes mentales (angl. mental maps, cf. entre autres Preston/Niedzielski 2000 ; Lameli/Purschke/Kehrein 2008 ; pour le français, cf. Kuiper 1999 ; Pustka 2010) : les informateurs étaient invités, d'une part, à indiquer les contours et à nommer les différentes aires de diffusion des variétés de français qu'ils pensaient être capables de distinguer sur une carte de leur région linguistique (France, Suisse romande ou Québec). D’autre part, ils avaient pour tâche d'entourer l'endroit où l'on parle, selon eux, le meilleur français à l'intérieur de cette même région. Les résultats de la question n'ont cependant pas été utilisés dans le cadre du présent ouvrage, notamment en raison des connaissances géographiques souvent lacunaires des informateurs, menant à certaines distorsions des résultats.

\subsubsection{Section 4 : représentations de la prononciation des présentateurs de télévision des régions étudiées}

Cette section concerne les représentations des informateurs des trois régions étudiées par rapport au rôle des présentateurs de télévision dans la définition de la norme de prononciation. Étant données les critiques formulées au Québec par certains linguistes considérant la prononciation de Radio-Canada comme trop proche de celle de la norme traditionnelle de référence pour représenter une norme de référence nationale, ${ }^{2}$ la section comprend deux questions touchant à la légitimité du modèle des présentateurs au sein des trois communautés.

Question 6.1 : Les présentateurs des journaux télévisés parisiens/suisses romands/québécois ont-ils un accent propre à Paris/à la Suisse romande/au Québec ? ( $\square$ Oui $\square$ Non)

Question 6.2 : Si oui, qu'est-ce qui les différencie des présentateurs de journaux télévisés d'autres pays/régions francophones?

La première de ces deux questions cherchait à mettre en évidence dans quelle mesure la prononciation des présentateurs de journaux télévisés de chacune des régions est considérée par les informateurs « non experts » comme une prononciation standard propre à leur région et différente de la prononciation d'autres régions francophones (cf. question 6.1). Les informateurs avaient la possibilité

2 Notons que ces critiques persistent malgré les résultats de différentes études sociolinguistiques présentées au chapitre 2.1.2.3.4 montrant la vaste acceptation de ce modèle de norme au sein de la population québécoise. 
d'y répondre par oui ou non et d'ajouter des exemples différenciant la prononciation des présentateurs de leur région de celle d'autres régions francophones (cf. question 6.2).

Question 7 : Selon vous, quel est le français / le français suisse romand / le français québécois le plus représentatif de l'ensemble de la France / de la Suisse romande / du Québec?

Possibilités de réponse : $\square$ le français parlé dans le journal télévisé, $\square$ le français utilisé dans une discussion entre des étudiants, $\square$ le français parlé dans la vie quotidienne à la campagne, $\square$ autre

La deuxième question (cf. question 7) cherchait, pour sa part, à vérifier le rôle de modèle de prononciation des présentateurs de télévision au sein de chacune des communautés et se rattache donc au même type que les questions proposées par Bouchard/Maurais $(2001,112)$ pour le Québec (cf. 2.2.1.5.2). Afin de pouvoir quantifier les résultats et les comparer à ceux des études préalables, les possibilités de réponses ont été présentées dans ce cas-ci sous forme de choix simple parmi trois possibilités de réponse illustrant différentes situations de communications. Par ailleurs, les informateurs avaient la possibilité de formuler librement une autre réponse si besoin était (réponse autre).

\subsubsection{Section 5 : attitudes face à la prononciation de chaque région dans une comparaison avec les prononciations d'autres régions francophones}

La dernière section de ce questionnaire touche aux attitudes des informateurs face aux différences de prestige manifeste et latent de la prononciation de leur région par rapport à celle d’autres régions.

Question 8 (Suisse romande, Québec) : Quand, à la télévision, vous regardez une publicité suisse / québécoise utilisant l'accent suisse romand / québécois, vous vous sentez :

Possibilités de réponse : ( $\square$ fier $\square$ amusé $\square$ gêné $\square$ agacé $\square$ indifférent)

Question 8 (Paris) : Quand, à la télévision, vous regardez une publicité utilisant un accent régional, vous vous sentez:

Possibilités de réponse : ( $\square$ charmé $\square$ amusé $\square$ agacé $\square$ indifférent)

La première question de cette section (cf. question 8), qui est également inspirée d'une question posée par Singy (1996, 286, question 5), ${ }^{3}$ visait à mettre en lumière les attitudes des informateurs face à l'utilisation de la prononciation de leur région dans des contextes de parole publique. Cette question avait donc pour objectif de

3 Question 5 du questionnaire de Singy : «Quand vous voyez une publicité française qui utilise

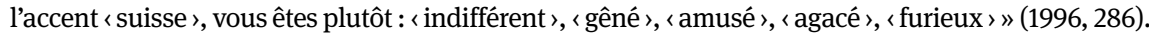


mettre en lumière une éventuelle insécurité linguistique chez les informateurs des variétés non dominantes suisse romande et québécoise. Dans le cas de Paris cependant, la question a été inversée afin de mettre en lumière la perspective contraire, supposée au vu des résultats publiés dans les précédents travaux (cf. 2.1.2.3.2) : celle de la sécurité linguistique, associée à des qualificatifs liés aux variétés dominantes. Les possibilités de réponse proposées aux informateurs parisiens ont par conséquent également été adaptées à cette perspective. Notons finalement qu'ici aussi, les possibilités de réponses ont été préétablies, pour des raisons de quantification.

Le thème de la sécurité/l'insécurité linguistique est abordé également dans les cinq questions suivantes : les trois premières touchent au rapport qu'entretiennent les informateurs face au prestige de leur prononciation par rapport à celui d'autres régions francophones et les suivantes à ces différences de prestige à l'intérieur de chacune des régions. Notons que les formulations de ces questions sont à nouveau reprises à Singy (1996, 286, questions 11-15). ${ }^{4}$

Question 9 (Suisse romande, Québec) : Face à un Français ayant la même profession, comment un Suisse romand / un Québécois se sent-il par rapport à sa façon de prononcer?

Question 9 (Paris, redoublée) :

- Face à un Suisse ayant la même profession, comment un Parisien se sent-il par rapport à sa façon de parler?

- Face à un Québécois ayant la même profession, comment un Parisien se sent-il par rapport à sa façon de parler?

Possibilités de réponse (pour toutes les questions) : $\square$ en situation d'égalité, $\square$ en situation d'infériorité, $\square$ en situation de supériorité

La première des cinq questions (cf. question 9) visait à mettre en lumière la différence de sécurité/d'insécurité linguistique des informateurs " périphériques » (Suisse romande, Québec) par rapport à celle des informateurs français. La question a été formulée avec l'ajout ayant la même profession afin que les informateurs ne puissent se référer qu'aux différences de prestige dues à l'origine géographique des locuteurs et non à leur origine sociale. Notons que dans le cas des Parisiens,

4 Questions 11-13 du questionnaire de Singy $(1996,286)$ : «Face à un Français ayant la même profession, un Romand se sent-il par rapport à sa façon de parler : » (11), « Face à un Belge ayant la même profession, un Romand se sent-il par rapport à sa façon de parler : » (12), " Face à un Lausannois ayant la même profession, un Payernois se sent-il par rapport à sa façon de parler : » (13) ; possibilités de réponse (pour les trois questions) : " en situation d'égalité », " d'infériorité » et « de supériorité ». Question 14 : «À votre avis, le jugement des Belges sur le parler des Vaudois est : »; possibilités de réponse : « le même », " plus critique que celui des Français », " moins critique ». Question $15:$ « Le jugement que portent les Lausannois sur le parler des autres Vaudois est plutôt : »; possibilités de réponse : « négatif », « positif », « ils ne portent pas de jugement ». 
la question a été inversée et redoublée afin de pouvoir confronter les attitudes qu'ils présentent face aux locuteurs québécois à celles qu'ils présentent face aux locuteurs suisses romands. J'ai, ici aussi, opté pour une réponse à choix simple parmi trois possibilités de réponses (en situation d'égalité, en situation d'infériorité, en situation de supériorité).

Question 10 (Suisse romande, Québec) : Face à un Suisse / à un Québécois ayant la même profession, comment un Québécois / un Suisse se sent-il par rapport à sa façon de parler?

Question 10 (Paris, redoublée) :

- Face à un Tourangeau ayant la même profession, comment un Parisien se sent-il par rapport à sa façon de parler?

- Face à un Toulousain ayant la même profession, comment un Parisien se sent-il par rapport à sa façon de parler?

Possibilités de réponse (pour toutes les questions) : $\square$ en situation d'égalité, $\square$ en situation d'infériorité, $\square$ en situation de supériorité

La deuxième de ces cinq questions (cf. question 10) aborde la même thématique, mais cette fois-ci, dans les cas de la Suisse romande et du Québec, dans le cadre d'une comparaison non plus avec des locuteurs parisiens, mais avec des locuteurs d'une autre variété « périphérique ». Dans le cas de Paris, la question a été redoublée afin découvrir les attitudes non seulement face à une variété "périphérique » interne à la France (la variété toulousaine), mais également face à l'autre variété de prestige française, celle de Tours (cf. 2.1.2.3.2). Notons que les possibilités de réponse proposées aux informateurs sont les mêmes que pour la question précédente (en situation d'égalité, en situation d'infériorité, en situation de supériorité).

Question 11 : À votre avis, le jugement des Suisses / des Québécois sur l'accent des Québécois / des Suisses est. . . ( $\square$. . . le même $\square$.. . plus critique que celui des Français $\square$. . . moins critique que celui des Français)

La troisième des cinq questions (cf. question 11) complète les deux précédentes en demandant explicitement aux informateurs d'estimer s'ils pensent que des locuteurs français jugeront leur accent plus négativement que des locuteurs d'autres « périphéries ». Comme cette question ne touche que les deux variétés "périphériques », elle n’a pas été utilisée dans le questionnaire parisien. Il s’agit, ici aussi pour des raisons de quantification, d'une question à choix simple parmi trois possibilités de réponses. 
Question 12 (Suisse) : Face à un Genevois ayant la même profession, comment un Neuchâtelois se sent-il par rapport à sa façon de parler?

Question 13 (Suisse) : Face à un Lausannois ayant la même profession, comment un Neuchâtelois se sent-il par rapport à sa façon de parler?

Question 12 (Québec) : Face à un Montréalais ayant la même profession, comment un Magnymontois se sent-il par rapport à sa façon de parler?

Question 13 (Québec) : Face à un Québécois de la ville de Québec ayant la même profession, comment un Magnymontois se sent-il par rapport à sa façon de parler?

Possibilités de réponse (pour toutes les questions) :

$\square$ en situation d'égalité, $\square$ en situation d'infériorité, $\square$ en situation de supériorité

Les deux dernières de ces cinq questions (cf. questions 12 et 13) reprennent la thématique des différences de prestige des deux précédentes, mais à l'intérieur des communautés étudiées. Ces questions n'ont, elles aussi, été posées qu'en Suisse romande et au Québec, étant donné que la question d'un modèle de référence interne à une région ne se pose pas réellement dans le cas de Paris. Elle ne se trouve donc pas dans le questionnaire parisien.

Ainsi, deux villes pouvant jouer un rôle de modèle de référence régional ont été respectivement prises en compte dans les questionnaires distribués en Suisse romande (Genève et Lausanne) et au Québec (Montréal et la ville de Québec). Le choix de ces quatre villes s'explique comme suit : Genève présente un statut supposé de standard régional en Suisse romande (cf. Pooley 2012 ; Thibault 1998 ; Racine/ Schwab/Detey 2013) et accueille le siège de la télévision de la RTS (Radio Télévision Suisse). Lausanne revêt une fonction de siège de la radio de cette même $R T S$, représente démographiquement la deuxième ville de Suisse romande $(\sim 135.000$ habitants, cf. Office fédéral de la statistique 2017) et a déjà été prise en compte par Singy $(1996,268)$, ce qui permet une comparaison de ses résultats avec les miens. Pour ce qui est du Québec, le choix de Montréal et de la ville de Québec se justifie par le découpage linguistique traditionnel du Québec en deux zones : l'Est, centré autour de la ville de Québec, et l'Ouest, autour de Montréal (cf. Poirier 1994 ; Verreault/ Lavoie 2004).

Quant aux villes de Neuchâtel et Montmagny, elles ont été prises en compte en tant que pôles opposés, en raison de leur éloignement géographique des zones au statut supposé de standard régional (Genève en Suisse, Montréal et Québec au Québec) et de leur poids démographique beaucoup plus faible que pour les quatre villes mentionnées plus haut (Neuchâtel : 34.000 habitants, cf. Office fédéral de la statistique 2017 ; Montmagny : 11.200 habitants, cf. Statistique Canada 2016). Notons finalement que les possibilités de réponses sont les mêmes que pour la question 10 (cf. supra) (en situation d'égalité, en situation d'infériorité, en situation de supériorité). 
Question 14 : Selon vous, quel accent de français devrait-on apprendre dans les cours de français langue étrangère (FLE) donnés en France / en Suisse romande / au Québec?

Possibilités de réponse (Paris) : $\square$ l'accent parlé dans les médias français, $\square$ l'accent parisien, $\square$ l'accent de Tours, $\square$ aucun accent/accent neutre, $\square$ peu importe, $\square$ autre

Possibilités de réponse (Suisse romande) : $\square$ l'accent genevois, $\square$ l'accent lausannois, $\square$ l'accent suisse romand parlé dans les médias, $\square$ l'accent parisien, $\square$ aucun accent/accent neutre, $\square$ peu importe, $\square$ autre

Possibilités de réponse (Québec) : $\square$ l'accent québécois de Montréal, $\square$ l'accent québécois de la Ville de Québec, $\square$ l'accent québécois parlé dans les médias, $\square$ l'accent parisien, $\square$ aucun accent/accent neutre, $\square$ peu importe, $\square$ autre

La question suivante (cf. question 14) porte sur le modèle de prononciation à enseigner dans les cours de FLE en immersion en France, en Suisse romande et au Québec. C'est en effet dans ce domaine que la question d'une norme de référence est particulièrement pertinente. Par ailleurs, les réponses auront tendance à être différentes dans un cadre d'immersion par rapport au cadre d'apprentissage traditionnel restreint à la classe de langue (cf. entre autres Regan/Howard/ Lemée 2009 ; Mougeon/Nadasdi/Rehner 2010 ; Regan 2013). Pour cette question également, j’ai opté pour une réponse à choix simple parmi plusieurs possibilités, en prenant en compte des accents pouvant éventuellement jouer un rôle dans la définition d'un standard régional de chacune des trois régions (cf. supra).

Question 15 (Suisse romande, Québec) : Comment les Parisiens perçoivent-ils la façon de prononcer des Suisses romands / des Québécois?

Question 16 (Suisse romande, Québec) : Comment les Parisiens perçoivent-ils leur propre façon de prononcer?

Les deux questions suivantes (cf. questions 15 et 16) visaient à découvrir explicitement les qualificatifs utilisés par les informateurs "périphériques » suisses romands et québécois pour décrire leur sentiment d'infériorité linguistique par rapport aux Parisiens. Afin d'éviter d'influencer les informateurs dans leur choix de ces qualificatifs, les questions sont ouvertes et sans possibilités de réponse préétablies, la catégorisation des données s'étant faite a posteriori.

Notons que, tout comme dans le cas des questions 11, 12 et 13, la présente question n'a été posée qu'en Suisse romande et au Québec, étant donné qu'elle porte sur le sentiment d'insécurité linguistique des locuteurs « périphériques ».

Question 17 : Lequel des accents suivants est selon vous...

Possibilités de réponse : $\square$ le plus beau, $\square$ le plus laid, $\square$ le plus sympathique, $\square$ le moins sympathique, $\square$ le plus sérieux, $\square$ le plus ridicule, $\square$ le plus raffiné, $\square$ le moins raffiné, $\square$ le plus correct, $\square$ le moins correct

Accents pris en compte (Paris) : parisien, méridional, tourangeau, suisse, belge, africain, québécois 
Accents pris en compte (Suisse romande) : parisien, méridional, québécois, belge, africain, genevois, lausannois

Accents pris en compte (Québec) : parisien, méridional, suisse, belge, africain, acadien, québécois de Montréal, québécois de Québec

Finalement, des qualificatifs préétablis faisant référence aux axes de la pleasantness et de la correctness de Preston (1999b, xxxiv) (cf. 2.1.2.3.2) ont été combinés dans une dernière question (cf. question 17) portant sur les attitudes des informateurs de chacune des trois régions face à différents accents. Lequel des accents suivants est selon vous... (possibilités de réponse : le plus beau - le plus laid, le plus sympathique - le moins sympathique pour l'axe de la pleasantness ; le plus sérieux - le plus ridicule, le plus raffiné - le moins raffiné, le plus correct - le moins correct pour l'axe de la correctness). Notons que les formulations de ces qualificatifs sont en partie inspirées de l'étude de Pustka (2010, 137). Les accents pris en compte incluent, pour la Suisse romande et le Québec, l'accent exogène considéré traditionnellement comme modèle de référence (parisien) et de potentiels modèles de prononciation internes à chacune de ces deux régions "périphériques » (accent québécois de Montréal et de Québec pour le Québec, accent genevois et lausannois pour la Suisse romande).

Notons que dans le cas du Québec, le choix des villes de Montréal et de Québec s'explique par le fait qu'il s'agit des deux principaux accents associés au Québec " urbain » et perçus positivement en termes normatifs dans l'étude de Remysen (2016b, 52-53). Ce choix a également permis d'étudier les différences de représentations des prononciations des deux grandes régions québécoises établies par Lavoie (1994), Bergeron (1995) et Verreault/Lavoie (2004) : la région de l'Est, qui est centrée autour de la ville de Québec, et celle de l'Ouest, centrée autour de la ville de Montréal. Dans le questionnaire destiné aux informateurs parisiens, ces différenciations internes à la Suisse et au Québec n'ont pas été faites, mais l'accent tourangeau a été ajouté en raison de son statut traditionnel de prononciation de prestige en France, à côté de celui de Paris (cf. 2.2.1.1).

\subsubsection{Informations sociodémographiques}

Dans un dernier temps, les informateurs ont été conviés à faire état d'une série de données sociodémographiques. Comme il s'agit d'une des parties délicates de tout questionnaire, elle a été, selon la règle évoquée par Hippler/Seidel (1985, 43), placée à la fin du questionnaire. Les informations récoltées comprennent non seulement les données sociodémographiques comprises traditionnellement dans de nombreuses études en sciences sociales (sexe, âge, lieu de naissance, domicile actuel et nombre d'années durant lesquelles les informateurs y ont vécu, niveau 
d'éducation, profession), mais également des informations nécessaires à l'interprétation des résultats touchant aux attitudes des informateurs suisses romands et québécois par rapport à la prononciation parisienne (éventuels séjours prolongés en France, stations de radio et chaînes de télévision - régionales et/ou françaises - écoutées et regardées par les informateurs québécois et suisses romands). Notons qu'en ce qui concerne le niveau d'éducation, le questionnaire a été adapté au système éducatif de chacune des régions (cf. annexes 1-3, section Questions sur l'informateur).

\section{Questions sur l'informateur}

$\square$ Femme $\quad \square$ Homme

Âge : ans

Où êtes-vous né ?

Où habitez-vous actuellement (1) et depuis combien de mois / combien d'années (2) ?

(1)

(2) Depuis

Êtes-vous déjà allé en France ?

$\square$ jamais

$\square$ pour des vacances

$\square$ pour y vivre (nombre d'années vécues en France : ans)

Quel est le dernier diplôme que vous avez obtenu ?

(primaire, secondaire, collégial (Cégep), universitaire (baccalauréat, maîtrise et doctorat))

Quelle est votre profession actuelle?

Origine du père :

Origine de la mère :

Quelle(s) chaîne(s) de radio écoutez-vous le plus souvent?

Quelle(s) chaîne(s) de télévision regardez-vous le plus souvent ?

Regardez-vous des chaînes de télévision françaises ? $\quad \square$ Oui $\square$ Non

Si oui, lesquelles?

Figure 3 : Questions sur l'informateur du questionnaire distribué au Québec (à titre d'exemple). 


\subsubsection{Sélection et recrutement des informateurs}

Dans de nombreuses études sociolinguistiques similaires à la présente enquête, la sélection d'informateurs se restreint à un échantillon spécifique de la population dans le cadre d'un échantillonnage dirigé. Cette méthode non probabiliste présente en effet l'avantage considérable de permettre de réunir un nombre élevé d'informateurs en peu de temps et de considérer l'enquête comme valable pour ce groupe d'auditeurs en particulier, étant donnée la concentration d'informateurs présentant un profil sociodémographique homogène (cf. Semaan 2010, 64). La méthode n'est cependant pas adéquate pour atteindre une certaine représentativité d'une communauté linguistique. L'autre pôle est celui de l'échantillonnage aléatoire, une méthode probabiliste qui permet d'obtenir la meilleure représentativité parmi les méthodes d'échantillonnage, mais qui ne peut être utilisée que dans le cadre d'enquêtes de grande envergure en raison du vaste échantillon nécessaire pour obtenir une certaine représentativité des différents groupes sociodémographiques (cf. Beaud 2010, 269-279).

Étant donné que la présente étude visait à obtenir des résultats les plus généralisables possible (cf. 3.2) sans pour autant disposer des ressources nécessaires pour obtenir une base de sondage suffisante à l'utilisation de l'échantillonnage aléatoire, les informateurs ont été choisis selon une méthode se situant entre les échantillonnages dirigés d'un groupe spécifique et les échantillonnages aléatoires : il s'agit de l'échantillonnage par quotas (cf. Beaud 2010, 267-269 ; cf. également Noelle 1963 ; Koolwijk 1974 ; Böltken 1976). Ce type d'échantillonnage s'effectue selon un nombre précis (un quota) et défini au préalable d'informateurs à atteindre pour des sous-populations, dans le but de pouvoir effectuer une estimation relativement fiable des résultats pour la population définie sur la base d'un nombre relativement restreint d'informateurs. Notons que cette méthode est courante dans le domaine des sciences sociales (cf. Koolwijk 1974, 81-99 ; Matalon 1988, 81), mais qu'elle est également controversée (cf. Weischer 2004, 140-141 ; Beaud 2010, 268-269) : puisque ce type d'échantillonnage est non probabiliste, c'est-à-dire que l'enquêteur a la possibilité de choisir ses informateurs lui-même avec la seule restriction d'obtenir des informateurs aux profils prédéfinis, le «tirage se fait accidentellement et non aléatoirement » (Beaud 2010, 268) à l'intérieur de chaque sous-groupe sociodémographique. Ainsi, cette méthode peut mener à des distorsions des résultats, de nombreux facteurs pouvant influencer les résultats : l'endroit ou le moment choisi dans la journée pourront par exemple favoriser certains profils d'enquêtés au détriment d'autres (cf. Beaud 2010, 269). Au sens strict du terme, cette méthode ne peut donc pas être considérée comme représentative et elle est principalement justifiable en invoquant des raisons pratiques : lorsque le chercheur peut s'at- 
tendre à un taux de réponses relativement faible, lorsque la population étudiée présente une répartition de profils sociodémographiques très déséquilibrée (cf. Diekmann ${ }^{6} 2012$, 393-394) ou encore lorsque les ressources à disposition exigent une certaine économie de travail (cf. Postlep 2010, 99). Malgré les inconvénients évoqués, soulignons que l'on peut tout de même " accorder une assez grande confiance aux enquêtes par quotas » (cf. Ardilly ${ }^{2} 2006,220$ ), étant donné que des études comparatives ont pu montrer une équivalence entre les performances des échantillonnages par quotas et celles des échantillonnages aléatoires lorsque les échantillons sont de petite taille (cf. Desabie 1966, 44 ; Ardilly 22006, 140), comme dans la présente étude.

Ainsi, l'échantillonnage s'est fait sur la base de quatre variables sociodémographiques : le sexe, l'âge, le niveau d'éducation et l'origine géographique des informateurs (cf. tableau 11). Les trois premières variables (sexe, âge et niveau d'éducation) ont été réparties de la façon suivante : la parité entre femmes (50\%) et hommes (50\%) a été respectée, la variable de l'âge a été répartie en trois catégories (20-39 ans, 40-59 ans, 60 ans et plus) et celle du niveau d'éducation en deux

Tableau 11 : Choix des informateurs par point d'enquête selon leur sexe, leur âge et leur niveau d'éducation.

\begin{tabular}{|c|c|c|c|c|c|}
\hline Sexe & & Âge & & Niveau d'éducation & \\
\hline \multirow[t]{6}{*}{ Femmes } & 12 & $20-39$ & 4 & Diplôme universitaire et diplôme de fin d'études secondaires & 2 \\
\hline & & & & Diplôme inférieur ou aucun diplôme & 2 \\
\hline & & $40-59$ & 4 & Diplôme universitaire et diplôme de fin d'études secondaires & 2 \\
\hline & & & & Diplôme inférieur ou aucun diplôme & 2 \\
\hline & & $60+$ & 4 & Diplôme universitaire et diplôme de fin d'études secondaires & 2 \\
\hline & & & & Diplôme inférieur ou aucun diplôme & 2 \\
\hline \multirow[t]{6}{*}{ Hommes } & 12 & $20-39$ & 4 & Diplôme universitaire et diplôme de fin d'études secondaires & 2 \\
\hline & & & & Diplôme inférieur ou aucun diplôme & 2 \\
\hline & & $40-59$ & 4 & Diplôme universitaire et diplôme de fin d'études secondaires & 2 \\
\hline & & & & Diplôme inférieur ou aucun diplôme & 2 \\
\hline & & $60+$ & 4 & Diplôme universitaire et diplôme de fin d'études secondaires & 2 \\
\hline & & & & Diplôme inférieur ou aucun diplôme & 2 \\
\hline Total & & & & & 24 \\
\hline
\end{tabular}


catégories (diplôme universitaire et diplôme de fin d'études secondaires selon la Classification Internationale Type de l'Éducation (CITE) ${ }^{5}$ vs. diplôme inférieur ou aucun diplôme). Notons que la répartition de ces niveaux d'éducation est bien entendu différente d'une région à l'autre : pour la France, le diplôme d'études secondaires pré-universitaires correspond au Baccalauréat, pour la Suisse à la Maturité et pour le Québec au Diplôme d'études collégiales (DEC) des programmes d'études pré-universitaires. Compte tenu de cette catégorisation et de l'objectif fixé d'un minimum de deux informateurs par sous-groupe sociodémographique (colonne de droite), le nombre d'informateurs s'élève à 24 par point d'enquête, donc 96 par région :

Le tableau 11 se lit de gauche à droite : chaque point d'enquête réunit 12 femmes et 12 hommes, ces deux catégories réunissant respectivement 4 informateurs de 20 à 39 ans, quatre informateurs de 40 à 59 ans et quatre informateurs de 60 ans et plus, ces quatre informateurs étant respectivement eux-mêmes répartis en deux niveaux d'éducation.

En ce qui concerne la variable de l'origine géographique des informateurs, quatre points d'enquête ont été retenus respectivement dans chacune des trois régions (Paris, Suisse romande, Québec).

\section{Paris}

Dans le cas de Paris, la répartition s'est faite principalement à travers une combinaison de deux critères : les couches sociales (favorisées et défavorisées) et la différence entre Paris intra-muros et extra-muros. Ainsi, le choix de points d'enquête s'est porté sur le $16^{\text {ème }}$ arrondissement (intra-muros, couches sociales plutôt favorisées), Neuilly-sur-Seine (extra-muros, couches sociales plutôt favorisées), le $19^{\text {ème }}$ arrondissement (intra-muros, couches sociales plutôt défavorisées) et la Courneuve (extra-muros, couches sociales plutôt défavorisées) (cf. figure 4). Cette répartition correspond également aux auto-représentations que donnent les Parisiens de leurs différents accents (cf. Pustka 2008, 244-245 ; cf. également chapitre 2.3.2), avec l'accent dit « snob » de la haute bourgeoisie du $16^{\text {ème }}$ arrondissement et de Neuilly-sur-Seine, l'accent dit « parigot » de la classe ouvrière des quartiers de Belleville et Ménilmontant (représenté par le $19^{\text {ème }}$ arrondissement) et l'accent dit « de banlieue » des personnes issues de l'immigration habitant dans les banlieues (représenté par la Courneuve).

5 Il s'agit de la classification des systèmes éducatifs de référence de l'UNESCO (cf. Institut de statistique de l'UNESCO 2011). 


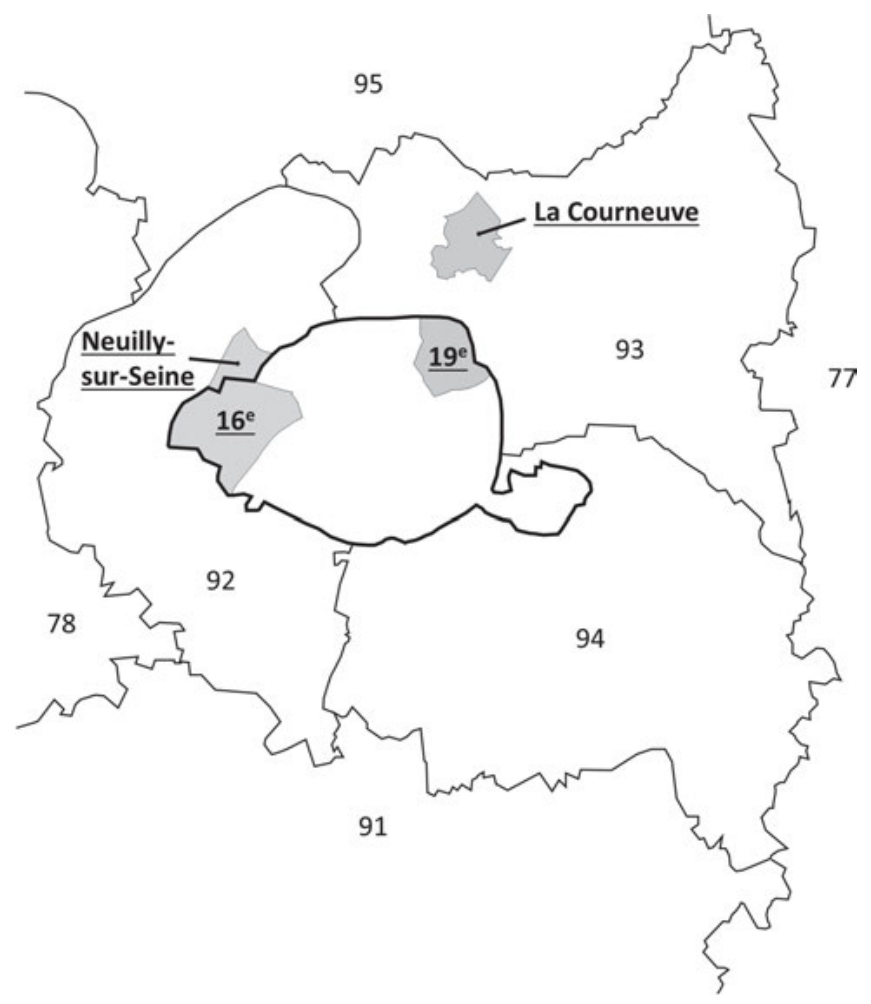

Figure 4 : Quatre points d'enquête retenus à Paris.

\section{Suisse romande}

Pour la Suisse romande, le choix des points d'enquête s'est fait sur la base de la répartition linguistique traditionnelle de cet espace en cantons, même si cette répartition ne correspond pas forcément à la réalité linguistique, le français parlé en Suisse romande étant hétérogène (cf. Andreassen/Maître/Racine 2010, 216) : ses particularismes s'étendent en effet sur une partie plus ou moins étendue du territoire (local, cantonal, supra-cantonal, etc.), débordent ou non des frontières politiques et se retrouvent ou non dans d'autres régions francophones (limitrophes ou non) (cf. Knecht 1979, 254-255 ; Kristol 1994, 239-241 ; Thibault 2000, 81-82 ; Andreassen/Maître/Racine 2010, 216). Ainsi, parmi les sept cantons formant la Suisse romande (quatre cantons officiellement unilingues : Genève, Jura, Neuchâtel, Vaud ; trois cantons officiellement bilingues : Berne, Fribourg, Valais), le choix s'est porté sur les cantons de Genève et de Neuchâtel. Le choix du canton de Genève s'explique par son statut supposé de standard régional en Suisse romande (cf. Racine/Schwab/Detey 2013) et par sa 
fonction de siège de la télévision de la Radio Télévision Suisse (RTS). Le canton de Neuchâtel a, pour sa part, été choisi en tant que canton unilingue formant un contraste avec celui de Genève, étant données sa localisation dans l'Est de la Suisse romande et ses frontières communes avec la Suisse alémanique. Afin de pouvoir étudier les disparités entre les villes et les zones rurales, le choix des points d'enquête s'est porté à l'intérieur de chacun des cantons sur une ville et une commune plus rurale (d'un point de vue démographique, cf. Office fédéral de la statistique 2017) : la ville de Genève ( 200.000 habitants) et la commune de Veyrier ( 11.500 habitants) dans le canton de Genève, la ville de Neuchâtel ( 34.000 habitants) et la commune de Boudry ( 6.000 habitants) dans le canton de Neuchâtel (cf. figure 5).

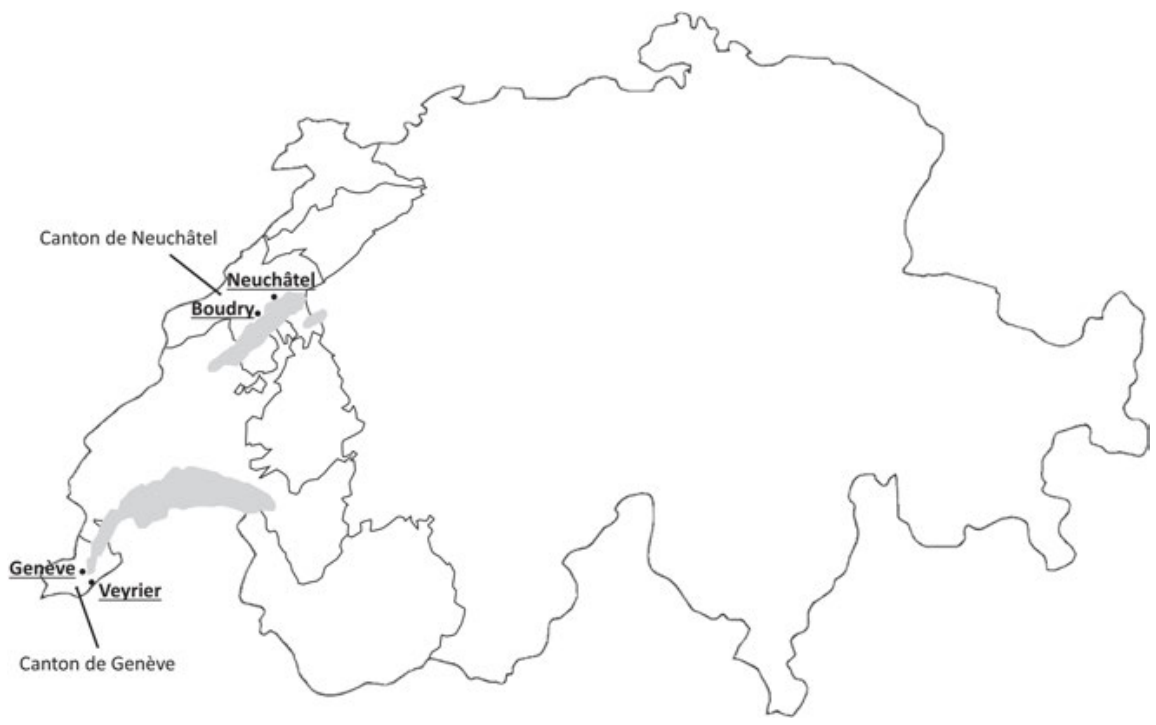

Figure 5 : Quatre points d'enquête retenus en Suisse romande.

\section{Québec}

Pour le Québec, c'est la séparation effectuée traditionnellement en dialectologie en deux grandes zones dialectales qui est à la base du choix des points d'enquête, avec l'Est du Québec centré autour de la ville de Québec et l'Ouest de la province centré autour de Montréal (cf. Verreault/Lavoie 2004 ; Poirier 1994), la zone du centre autour de Trois-Rivières ayant un statut ambigu et étant considérée comme une aire de transition (cf. Lavoie/Verreault 1999). Sur cette base dialectologique, le choix des points d'enquête s'est porté sur une grande ville 
et une petite ville rurale (à nouveau d'un point de vue démographique, cf. Statistique Canada 2016) dans chaque zone : la ville de Montréal ( 1,70 millions d'habitants) et celle de Lachute ( 12.800 habitants) dans la zone ouest, la ville de Québec ( $\sim 531.000$ habitants) et celle de Montmagny ( $\sim 11.200$ habitants) dans la zone est (cf. figure 6).

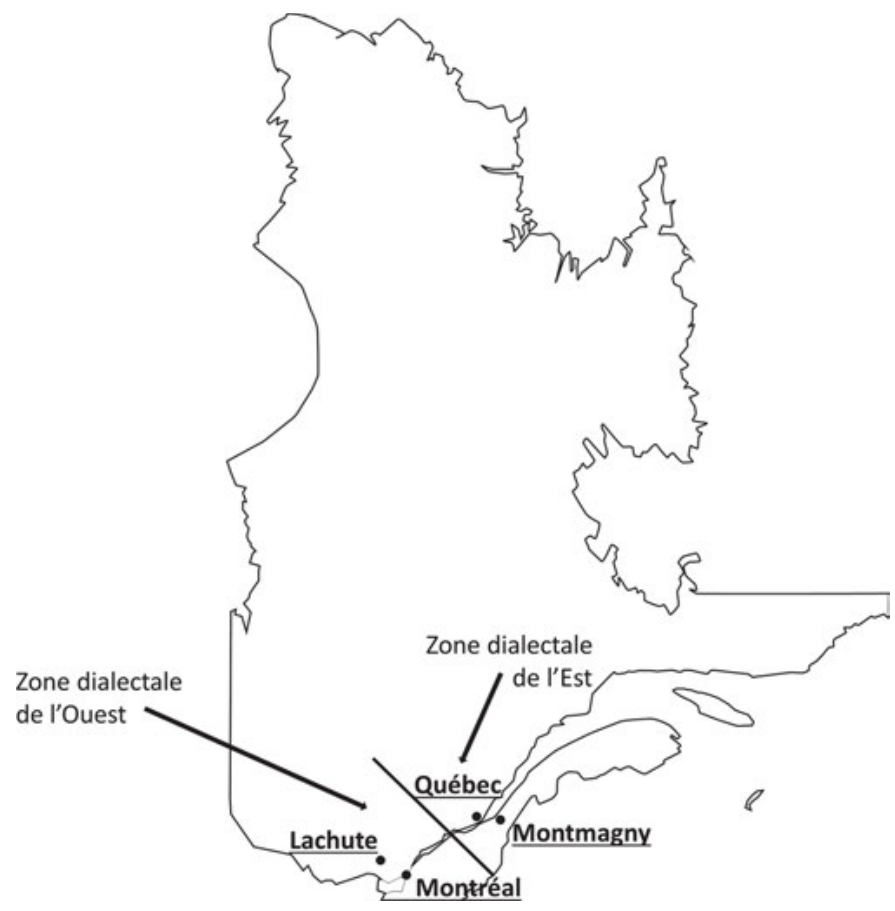

Figure 6 : Quatre points d'enquête retenus au Québec.

Ainsi, les questionnaires de 24 informateurs aux profils présentés dans le tableau 11 ont été retenus pour chacun de ces 12 points d'enquête ( 4 par région) : ceci correspond à 96 informateurs dans chacune des trois régions et à 288 informateurs au total. Ces informateurs devaient avoir vécu la majeure partie des dix dernières années - selon le cas présenté plus haut - dans le quartier, la commune, la ville ou l'agglomération urbaine en question et avoir au minimum un parent (père et/ ou mère) ayant vécu la plus grande partie de son enfance dans cet endroit. Tous les auditeurs n'entrant pas dans ces critères ont ainsi été exclus du traitement des données. 
Quant au recrutement concret des informateurs (Paris : septembre-octobre 2015 ; Suisse romande : juillet-août 2015 ; Québec : mars-avril 2015), ${ }^{6}$ il s'est fait sur la base de la méthode d'échantillonnage par quotas utilisée par Postlep (2010, 99) : dans un premier temps, la sélection des informateurs s'est faite de façon aléatoire et rapide, principalement dans les universités et les bibliothèques, dans les cafés et les bars ainsi que directement dans les rues. Une fois plusieurs quotas remplis ou lors de l'apparition d'un excédent de certains profils, la recherche s'est faite de façon ciblée, afin de pouvoir trouver des informateurs présentant les profils manquants et remplir également ces quotas. Après avoir été recrutés, les informateurs ont reçu de ma part une brève introduction destinée à leur expliquer la tâche à accomplir et son contexte, avant de procéder au remplissage du questionnaire écrit, une tâche qui a pris entre 20 et 30 minutes par informateur.

\subsubsection{Protocole d'analyse}

En préparation aux analyses statistiques des données des questionnaires, ces données ont tout d'abord été transférées manuellement dans une banque de données structurée à l'aide du logiciel Excel. Notons que les réponses ouvertes ont été catégorisées et quantifiées auparavant de façon manuelle et que la première question de chaque questionnaire (Quels accents de français connaissez-vous dans le monde ?) n'a pas été exploitée dans l'analyse des données, étant donné qu'il s'agissait d'une question d'introduction sans véritable valeur substantielle pour le sujet de recherche du présent ouvrage.

Les analyses statistiques ont ensuite été effectuées à l'aide du logiciel libre $R$. Chaque question a été soumise à une analyse de régression logistique afin de mesurer la signification statistique des résultats et de mettre en lumière les facteurs externes ayant influencé ces résultats. Pour chacune des questions, les possibilités de réponse ont été utilisées en tant que variable dépendante et les données sociodémographiques (sexe des locuteurs, âge, points d'enquête, niveau d'éducation, consommation régulière de médias audiovisuels français) en tant

6 Notons que le recrutement des informateurs à Paris s'est fait lors d'un séjour de recherche à l'Université Paris Ouest Nanterre La Défense financé entre autres par le programme ERASMUS + de mobilité d'enseignants en Europe. Le recrutement réalisé en Suisse été accompli à l'occasion d'un séjour de recherche à l'École de langue et de civilisation françaises de l'Université de Genève et avec le soutien financier de la Philologisch-Kulturwissenschaftliche Fakultät de l'Université de Vienne. Finalement, le séjour au Québec s'est déroulé dans le cadre d'un stage de recherche à l'Université Laval financé à l'aide d'une bourse de recherche de l'Université de Vienne (Kurzfristiges wissenschaftliches Auslandsstipendium). 
que variables indépendantes. Notons ce faisant que les catégories de réponses ne présentant que trois occurrences ou moins ont été systématiquement supprimées des données brutes avant l'application des modèles de régression, car certains d'entre eux requièrent un nombre minimum d'observations.

Selon le type de variable dépendante (binaire, qualitative à trois modalités ou plus, qualitative à trois modalités ou plus et ordonnée hiérarchiquement), un modèle de régression logistique différent a été appliqué aux données. Signalons que dans plusieurs cas, le modèle de régression qui avait été prévu initialement par le type de question a dû être remplacé par un autre modèle en raison du nombre trop faible de réponses dans une ou plusieurs des catégories (p. ex. dans le cas de la question 9 pour la Suisse romande, où le modèle de régression binomiale a été finalement substitué à la régression logistique ordinale prévue, étant donné que deux catégories ont été supprimées en raison du trop faible nombre de réponses :

- Dans le cas d'une variable dépendante binaire (c'est-à-dire de type « oui » ou « non »; questions 3.2 dans le cas de Paris ; questions 3.1, 4.1, 6.1 et 9 dans le cas de la Suisse romande ; questions 3.1, 4.1, 6.1, 9 et 10 dans le cas du Québec ; cf. annexes 1-3), une régression logistique binomiale a été appliquée aux données. Il s'agit, sous $R$, d'un modèle linéaire généralisé glm avec réponse binomiale et lien logit.

- Dans le cas d'une variable dépendante qualitative à trois modalités ou plus non ordonnée hiérarchiquement (questions 2, 3.1, 5, 6.1, 7, 8, 9.1, 9.2, 10.2, 14, 17 dans le cas de Paris ; questions 2, 3.2, 5, 7, 8, 12, 14, 17 dans le cas de la Suisse romande ; questions 2, 3.2, 5, 7, 8, 14, 17 dans le cas du Québec, cf. annexe 1-3), c'est une régression logistique multinomiale qui a été appliquée aux données. Pour ce faire, j'ai utilisé la fonction multinom de l'extension nnet sous $R$.

- Dans le cas d'une variable dépendante à trois modalités ou plus ordonnée hiérarchiquement (questions 10.1 dans le cas de Paris ; questions 10, 11, 13 dans le cas de la Suisse romande ; questions 11, 12, 13 dans le cas du Québec, cf. annexes 1-3), le choix s'est porté sur un modèle de régression logistique ordinale. À cet effet, j'ai utilisé la fonction polr de l'extension MASS sour R.

\subsection{Productions : analyse de voyelles produites par des locuteurs-modèles}

La deuxième méthode utilisée dans le présent ouvrage concerne les productions. Il s'agit d'une analyse de voyelles orales et nasales produites par des locuteursmodèles m'ayant permis de dégager les principales voyelles appartenant potentiellement aux trois normes étudiées. La présentation de cette méthode sera divisée en 
deux parties : dans un premier temps, je présenterai la collecte des données (cf. 3.4.1) et, dans un deuxième temps, les analyses des voyelles orales et nasales (cf. 3.4.2).

\subsubsection{Collecte des données}

La présentation de la collecte des données de production des locuteurs-modèles, à laquelle j'ai procédé en 2014 (octobre-novembre 2014 : Suisse romande) et 2015 (mars-avril 2015 : Québec, septembre 2015 : Paris), se fera en deux temps : j’aborderai, premièrement, les questions de la sélection et du recrutement des locuteursmodèles (cf. 3.4.1.1) et présenterai, par la suite, la question des enregistrements effectués avec ces locuteurs et du contenu de ces enregistrements (cf. 3.4.1.2).

\subsubsection{Sélection et recrutement des locuteurs-modèles}

Comme mentionné dans l'état de l'art, le présent ouvrage propose une approche de la norme de prononciation sur la base des productions de locuteurs-modèles (cf. Maurais 1985, 71 ; Léon 1994, 404 ; Reinke 2004, 14-17), des professionnels de la parole - en général des présentateurs de journaux radiophoniques ou télévisés - servant de modèle à leur (sous-)communauté linguistique. Notons qu'il faut cependant nuancer ce terme de présentateurs de journaux télévisés : si, il y a quelques décennies, de nombreuses chaînes de télévision engageaient des annonceurs (également appelés speakers en France et en Suisse romande) chargés de lire le texte des informations à l'antenne sans en avoir préparé le contenu, les différences entre présentateurs, journalistes et reporters se sont quasiment estompées dans les trois régions étudiées. Ainsi, les employés des chaînes publiques de ces trois régions sont formés dans chacun de ces domaines et écrivent en règle générale eux-mêmes les textes qu'ils présentent. Il est donc préférable d'utiliser le terme de journalistes-présentateurs et non de présentateurs (cf. Chalier 2020a, 96 ; cf. également Pustka/Chalier/Jansen 2017, 105-106 ; Chalier 2019, 428).

Soulignons, par ailleurs, que la formation des journalistes-présentateurs dans le domaine de la prononciation se limite en règle générale à la pose de voix, les questions d'accent n'étant que rarement abordées (cf. corpus Chalier 2015 ; cf. également Pustka/Chalier/Jansen 2017, 105-106 ; Chalier 2019, 428 ; Chalier 2020a, 96). L'usage linguistique homogène observable à l'antenne des stations de radio et des chaînes de télévision des trois régions analysées est en général expliqué par ces locuteurs par ce qu'ils nomment le « mimétisme », c'est-à-dire l'accommodation linguistique entre les différents journalistes-présentateurs de ces médias audiovisuels (cf. Pustka/Chalier/Jansen 2017, 105). De nombreux autres exemples peuvent être trouvés dans les entretiens guidés du corpus (cf. 3.4.1.2), dont les suivants : 
« M6 a impulsé [. . .] aussi une tonalité très particulière, et ensuite, ça se reporte sur les autres chaînes, et [. . .] tout le monde fait un peu la même chose. C’est le phénomène [. . .] d'identification, c'est le mimétisme aussi [...]» (locuteur cqTbs1).

« [ . . ] et puis vraiment, ou ils ont fait du mimétisme par rapport aux gens qui sont à l'antenne, ou on les fait travailler, travailler, travailler à l'antenne » (locuteur cqTsb1).

« [ . . . et puis je pense qu’après, il y a une espèce de [. . . mimétisme [. . .] (locuteur cmTad1).

(Corpus Chalier 2015)

Notons que l'étiquetage de ces locuteurs (cqTbs1, cqTsb1, cmTad1) ainsi que de tous les locuteurs du corpus (cf. tableaux 12, 13 et 14) suit les conventions du protocole du programme Phonologie du Français Contemporain PFC (cf. Durand/ Laks/Lyche 2002 ; 2009 ; Detey et al. 2016), mais les modifie sur deux points : le type de média dans lequel travaillent les locuteurs (radio ou télévision) a été ajouté et l'indice du point d'enquête (position 4 dans le protocole PFC) supprimé. Ainsi, dans le présent corpus, la première lettre indique le pays dans lequel a été effectué l'enregistrement ( $f$ pour France, $s$ pour Suisse et $c$ pour Canada), la deuxième est l'initiale de la ville ( $p$ pour Paris, $l$ pour Lausanne, $g$ pour Genève, $q$ pour Québec, $m$ pour Montréal et $o$ pour Ottawa) et la troisième (en majuscule) précise le type de média ( $R$ pour radio et $T$ pour télévision). Suivent ensuite les initiales des témoins en positions 5 et 6 ainsi qu'un chiffre en position 7 permettant de distinguer les locuteurs présentant les mêmes initiales.

La sélection de ces journalistes-présentateurs s'est faite sur la base de plusieurs critères sociodémographiques : le premier de ces critères était bien entendu leur fonction de journalistes-présentateurs au sein d'une des stations de radio ou chaînes de télévision de leur région. Il s'agit dans la grande majorité des cas de chaînes publiques, mis à part deux journalistes-présentateurs de radio de Paris, qui travaillent l'un et l'autre pour une station privée (BFM Radio, Europe 1 ; cf. tableau 12). Par ailleurs, étant donné que la recherche sur le modèle de prononciation des professionnels de la parole fait état aussi bien d'un modèle des locuteurs de la radio (cf. Léon 1966 ; 1968) que de ceux de la télévision (cf. Cajolet-Laganière/Martel 1995 ; Bouchard/Maurais 2001) (cf. 2.2.1.3), j’ai veillé à une répartition égale des journalistes-présentateurs dans chacun des média (radio et de télévision). Ainsi, 10 journalistes-présentateurs de télévision et 10 journalistes-présentateurs de radio ont été pris en compte dans chacun des trois sous-corpus, pour un total de 60 informateurs. En outre, étant donné que chacune des chaînes présente un certain pourcentage de journalistes-présentateurs originaires d'une autre communauté francophone, et de manière similaire au choix des informateurs ayant répondu au questionnaire écrit (cf. 3.3.2), le choix de ces locuteurs s'est porté uniquement sur les locuteurs natifs de chacune des régions. Ainsi, les journalistes-présentateurs recrutés, de même qu'un de leurs parents 
(mère et/ou père) au moins, devaient avoir vécu la majeure partie de leur enfance respectivement en France septentrionale, en Suisse romande ou au Québec, tous les locuteurs ne répondant pas à ces critères ayant été exclus du traitement des données. Finalement, j'ai veillé dans chaque sous-corpus à une répartition égale des sexes (dans l'idéal 50\% de femmes et 50\% d'hommes), qui n'a pas cependant pas toujours pu être respectée en raison du faible nombre de journalistesprésentateurs disponibles dans chaque région et du déséquilibre entre les sexes encore présent dans cette profession (cf. tableaux 12, 13 et 14).

\section{Paris}

À Paris, le recrutement s'est fait, pour la radio, au sein de plusieurs des stations publiques de Radio France (France Inter, France Info et France Culture) et, dans le cas de deux locuteurs, dans deux stations privées (BFM Radio et Europe 1). Pour la télévision, le recrutement a été effectué auprès des chaînes publiques France 2, France 3 Paris Île-de-France et TV5 Monde (cf. tableau 12).

Tableau 12 : Journalistes-présentateurs recrutés à Paris.

\begin{tabular}{|c|c|c|c|}
\hline Locuteur & Média & Chaîne & Sexe \\
\hline fpRrl1 & \multirow[t]{10}{*}{ Radio } & France Inter & Homme \\
\hline fpRom1 & & BFM Radio & Homme \\
\hline fpRsp1 & & France Inter & Homme \\
\hline fpRjo1 & & France Info & Homme \\
\hline fpRmp1 & & France Inter & Homme \\
\hline $\mathrm{fpRbd} 1$ & & France Info, France culture, France Inter & Homme \\
\hline fpRff1 & & Europe 1 & Homme \\
\hline fpRaa1 & & France Inter & Femme \\
\hline fpRms1 & & France Info & Femme \\
\hline fpRml1 & & France Inter & Femme \\
\hline fpTjc1 & \multirow[t]{10}{*}{ Télévision } & France 3 Paris Île-de-France & Homme \\
\hline fpTav1 & & France 2 & Homme \\
\hline fpTyk1 & & TV5 Monde & Homme \\
\hline fpTdd1 & & TV5 Monde & Homme \\
\hline fpTaf1 & & TV5 Monde & Homme \\
\hline fpTim1 & & TV5 Monde & Femme \\
\hline fpTms1 & & France 2 & Femme \\
\hline fpTim2 & & TV5 Monde & Femme \\
\hline fpTmt1 & & France 3 Paris Ile-de-France & Femme \\
\hline fpTmb1 & & France 2 & Femme \\
\hline
\end{tabular}




\section{Suisse romande}

En Suisse romande, le recrutement s'est fait aussi bien pour la télévision que pour la radio au sein de la Radio Télévision Suisse (RTS), une unité d'entreprise de la Société suisse de radiodiffusion et télévision : les journalistes-présentateurs de radio ont été recrutés dans la division des stations de radio de la $R T S$, qui a son siège à Lausanne, alors que les journalistes-présentateurs de télévision ont été recrutés, pour leur part, au sein de la division des chaînes de télévision de la RTS, qui a son siège à Genève. En ce qui concerne la radio, tous les journalistes-présentateurs travaillaient au moment des enregistrements pour la principale station publique RTS La Première. Pour ce qui est de la télévision, les journalistes-présentateurs travaillaient tous pour la RTS un et la RTS deux (cf. tableau 13).

Tableau 13 : Journalistes-présentateurs recrutés en Suisse romande.

\begin{tabular}{|c|c|c|c|c|}
\hline Locuteur & Ville & Média & Chaîne & Sexe \\
\hline slRsd1 & Lausanne & Radio & RTS La Première & Homme \\
\hline slRtc1 & & & & Homme \\
\hline slRjb1 & & & & Homme \\
\hline slRsm1 & & & & Homme \\
\hline slReg1 & & & & Homme \\
\hline slRad1 & & & & Homme \\
\hline slRcm1 & & & & Femme \\
\hline slRhk1 & & & & Femme \\
\hline slRas1 & & & & Femme \\
\hline slRlg1 & & & & Femme \\
\hline sgTml1 & Genève & Télévision & RTS un, RTS deux & Homme \\
\hline sgTlm1 & & & & Homme \\
\hline sgTba1 & & & & Homme \\
\hline sgTeb1 & & & & Homme \\
\hline sgTdr1 & & & & Homme \\
\hline sgTmp1 & & & & Femme \\
\hline sgTmm1 & & & & Femme \\
\hline$\overline{\text { sgTim } 1}$ & & & & Femme \\
\hline sgTrj1 & & & & Femme \\
\hline $\operatorname{sgTfd} 1^{7}$ & & & & Femme \\
\hline
\end{tabular}

7 Précisons qu'en raison d'un manque de données comparables, la dernière locutrice du sous-corpus suisse est une journaliste-présentatrice ayant travaillé aussi bien pour la division des stations de radio que pour celle des chaînes de télévision de la RTS, mais spécialisée dans le domaine de la radio. 


\section{Québec}

Au Québec, finalement, suivant les résultats de Gendron (1990), Cajolet-Laganière/ Martel (1995), Cox (1998), Martel (2001) et Corbeil (2007) montrant que le modèle de prononciation québécois semble se trouver dans l'usage des journalistesprésentateurs des journaux télévisés de la Société Radio-Canada (cf. 2.2.1.5.2), tous les locuteurs ont été recrutés au sein des chaînes de Radio-Canada : les journalistes-présentateurs de radio contactés sont en poste dans les antennes de la station de Radio-Canada ICI Radio-Canada Première à Montréal et à Québec, alors que les journalistes-présentateurs de télévision ont, pour leur part, été recrutés au sein des antennes de Montréal, de la ville de Québec et (pour une locutrice) d'Ottawa du réseau de télévision publique ICI Radio-Canada Télé. Notons que Montréal est le siège d'ICI Radio-Canada Première et d'ICIRadio-Canada Télé, alors que les autres antennes (dont Québec et Ottawa) sont considérées comme régionales (cf. tableau 14).

Tableau 14 : Journalistes-présentateurs recrutés au Québec.

\begin{tabular}{|c|c|c|c|c|}
\hline Locuteur & Ville & Média & Chaîne & Sexe \\
\hline cqRgl1 & Québec & Radio & ICI Radio-Canada & Homme \\
\hline cqRpa1 & Québec & & Première & Homme \\
\hline cqRcb1 & Québec & & & Homme \\
\hline $\mathrm{cmRfb} 1$ & Montréal & & & Homme \\
\hline cmRbl1 & Montréal & & & Homme \\
\hline cqRcl1 & Québec & & & Femme \\
\hline cqRsb1 & Québec & & & Femme \\
\hline cmRjp1 & Montréal & & & Femme \\
\hline cmRgl1 & Montréal & & & Femme \\
\hline coRmg1 & Ottawa & & & Femme \\
\hline cqTbs1 & Québec & Télévision & ICI Radio-Canada Télé & Homme \\
\hline cqTsb1 & Québec & & & Homme \\
\hline cmTjc1 & Montréal & & & Homme \\
\hline $\mathrm{cmTfc1}$ & Montréal & & & Homme \\
\hline $\mathrm{cmTmm} 1$ & Montréal & & & Homme \\
\hline cmTad1 & Montréal & & & Homme \\
\hline cmTvm1 & Montréal & & & Homme \\
\hline cmTga1 & Montréal & & & Femme \\
\hline cmTcb1 & Montréal & & & Femme \\
\hline $\mathrm{cmTfb} 1$ & Montréal & & & Femme \\
\hline
\end{tabular}




\subsubsection{Enregistrements des productions des locuteurs-modèles}

Le protocole d'enregistrement des locuteurs-modèles est une version modifiée $\mathrm{du}$ protocole du programme Phonologie du Français Contemporain PFC (cf. Durand/Laks/Lyche 2002 ; 2009 ; Detey et al. 2016). Trois des quatre situations de communication prévues par le protocole PFC ont été prises en compte lors des enregistrements : une modalité ouverte de la parole spontanée (un entretien guidé) et deux modalités fermées (un texte lu et une liste de mots). Notons que la liste de mots a été complétée par une liste supplémentaire propre au projet, cf. infra).

L'entretien guidé effectué avec les locuteurs-modèles a duré de 15 à 30 minutes. Il a été structuré selon deux axes thématiques : dans un premier temps, les locuteurs étaient invités à aborder leur formation (cursus universitaire, études de journalisme) et leur cursus professionnel en tant que journalistes (expériences professionnelles à l'étranger, notamment en France). Une question portait également sur leur éventuelle formation en prononciation suivie durant ce cursus (cours d'orthophonie avec éventuelles corrections de la prononciation, conditions préalables au travail en tant que présentateur des informations, rôle de l'accent dans la formation des présentateurs). Dans un deuxième temps, l'entretien portait - de manière parallèle aux questionnaires écrits (cf. 3.3) - sur leurs auto-représentations de la prononciation de leur région ainsi que sur leurs hétéro-représentations de la norme de prononciation traditionnelle et des autres prononciations francophones. Ce faisant, la structure des questionnaires écrits a été reprise, avec des sections thématiques touchant aux hétéro-représentations des accents de la francophonie et aux auto-représentations de la prononciation de la région d'origine des locuteurs et des différences de prononciation à l'intérieur de cette région. Afin de prendre en compte le degré de loquacité des enquêtés, le protocole de l'entretien été structuré sur la base de questions posées à tous les informateurs et de questions facultatives complémentaires (cf. annexes 4-6).

Le texte lu, pour sa part, est tiré du corpus PFC (398 mots, comptage Word). Il a été complété par l'ajout de 111 mots (comptage Word) développé initialement pour les enquêtes PFC suisses et belges (cf. Andreassen/Maître/Racine 2010 ; Andreassen/ Racine 2012), pour un total de 510 mots et une durée moyenne de 3 minutes par locuteur (cf. annexe 7). Il s'agit donc ici d'une situation de communication d'écrit oralisé, c'est-à-dire, selon le modèle de Koch/Oesterreicher ('2011), d'un écrit médial (graphie) et conceptionnel (distance communicative) transféré dans le médium phonique. Selon l'effet Buben (cf. Buben 1935), ou spelling pronunciation en anglais, une influence de la graphie sur la prononciation des journalistes devrait donc être observable, de manière similaire à la situation de lecture des informations sur un prompteur et des voix off à partir d'un texte dont les journalistes-présentateurs ont l'habitude. Notons que, contrairement à l'étude de Pustka/Chalier/Jansen (2017, 106), 
je n'ai pas pris en compte les présentations concrètes de journaux télévisés (ou voix off) de ces mêmes présentateurs dans les médias (radio et télévision), étant donné que pour les analyses acoustiques et les tests de perception, il était nécessaire de disposer de données les plus comparables possible.

La dernière tâche effectuée par les journalistes-présentateurs était une lecture de deux listes de mots. La première (cf. annexe 8), tirée du projet PFC et comptant 94 mots, a initialement été conçue par les responsables du programme PFC pour tester l'existence ou l'absence de toute une série de traits de prononciation du français (cf. entre autres Durand/Laks/Lyche 2002 ; 2009 ; Detey et al. 2016). Elle se prête bien à l'étude d'oppositions phonologiques et phonétiques de voyelles orales et nasales, car elle contient plusieurs paires minimales réparties dans un ordre aléatoire parmi les 84 premiers mots, alors que les 10 derniers mots présentent cinq paires minimales explicites, les deux mots de chaque paire minimale étant lus directement l'un après l'autre (p. ex. patte $\sim$ pâte, brin brun). De manière complémentaire, une deuxième liste de mots (cf. « liste de mots complémentaire », annexe 9) a été mise en place afin de pouvoir tester les traits de potentielles normes suisse romande et québécoise présentés dans les tableaux 7 et 8 ne se trouvant pas dans la liste PFC. ${ }^{8}$ Cette liste a été créée sur la base de mots extraits, d'une part, de la liste élaborée initialement pour les enquêtes PFC suisses et belges (cf. Andreassen/Maître/Racine 2010 ; Andreassen/Racine 2012 ; cf. tableau 15) et, d'autre part, de celle développée pour les enquêtes québécoises (cf. Côté 2012 ; cf. tableau 16). Soulignons que, dans l'optique d'études à venir, la liste complémentaire comprend également une série de mots se prêtant à l'analyse de traits consonantiques des normes suisses romandes potentielles (cf. section « consonnes » du tableau 15).

Notons finalement qu'à la fin de l'enregistrement de chacun des locuteursmodèles, ces derniers ont été invités à signer un consentement de participation repris en partie du projet PFC, mais adapté aux besoins de mes enquêtes. La garantie d'anonymat du consentement du projet PFC n'aurait en effet pas été suffisante à mes informateurs, étant donné qu'il s'agit de journalistes-présentateurs dont la voix est souvent connue du grand public. Ainsi, j'ai donné la possibilité aux informateurs d'autoriser ou de refuser la publication de chaque partie de l'enregistrement (entretiens guidés, lecture du texte, lecture des listes de mots) de façon séparée. De cette façon, les informateurs avaient la possibilité de refuser la publication de l'entretien guidé (qui, en tant que conversation, peut contenir des

8 Notons que Paris n’a pas été pris en compte ici, étant donné que les traits d'une potentielle norme de prononciation parisienne peuvent tous être testés sur la base de la liste PFC. Un aperçu de ces traits pourra être trouvé dans le tableau 6 . 
Tableau 15 : Traits suisses romands testés dans la liste de mots complémentaire (en référence à Andreassen/Maître/Racine 2010 ; Andreassen/Racine 2012).

\section{Traits suisses romands testés dans la liste de mots complémentaire}

\begin{tabular}{|c|c|c|}
\hline \multicolumn{3}{|c|}{ Voyelles } \\
\hline & Traits & Exemples \\
\hline \multirow[t]{4}{*}{$\begin{array}{l}\text { Oppositions } \\
\text { quantitatives }\end{array}$} & en syllabe finale fermée & 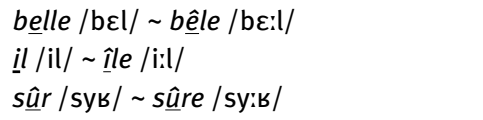 \\
\hline & $\begin{array}{l}\text { en syllabe finale fermée + différence } \\
\text { de timbre (voyelles de grande } \\
\text { aperture) }\end{array}$ & salle /sal/ sale /sa:l/ \\
\hline & $\begin{array}{l}\text { en syllabe finale ouverte } \\
\text { (+ éventuelle diphtongaison) }\end{array}$ & $\begin{array}{l}\text { ami /ami/ amie /ami:/ } \\
\text { venu /vəny/ venue /vəny:/ } \\
\text { penser /pãse/ pensée /pãse:/ } \\
\text { bleu /blø/ bleue /blø:/ } \\
\text { plaît /ple/ plaie /ple:/ } \\
\text { bout /bu/ boue /bu:/ } \\
\text { réseau / bezo/ zoo /zo:/ }\end{array}$ \\
\hline & & $a m \underline{i} / \mathrm{ami} / \sim a m \underline{i e} / \mathrm{ami}^{\mathrm{j} /}$ \\
\hline \multicolumn{2}{|c|}{$\begin{array}{l}\text { Opposition /e/ : / } \varepsilon / \text { en syllabe finale ouverte } \\
\text { (distinction de certains temps) }\end{array}$} & je pourrai /e/ je pourrais / $\varepsilon /$ \\
\hline \multicolumn{3}{|c|}{ Consonnes } \\
\hline & Traits & Exemples \\
\hline \multicolumn{2}{|c|}{ (Non-)prononciation de certaines consonnes finales } & 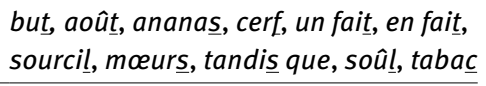 \\
\hline \multicolumn{2}{|c|}{ Assourdissement du / $\mathbf{~ / ~ f i n a l ~}$} & bar $[\mathrm{ba}: \mathrm{R}] \sim[\mathrm{ba}: \mathrm{R}]$ \\
\hline \multicolumn{2}{|c|}{ Fricative [x] et affriquée [kx] } & $\begin{array}{l}\text { Bach }[\text { bak] } \sim \text { [bax] } \\
\text { stöck }[\text { stœk }] \sim[\text { stœk] ( [jtœk }])\end{array}$ \\
\hline \multicolumn{2}{|c|}{ Palatalisation de certaines occlusives } & $\begin{array}{l}\text { quatre [katk] [k'ats] } \\
\text { époque [epэk'] }\end{array}$ \\
\hline
\end{tabular}

Tableau 16 : Traits québécois testés dans la liste de mots complémentaire (en référence à Côté 2012).

\section{Traits québécois testés dans la liste de mots complémentaire}

\begin{tabular}{|c|c|}
\hline Opposition quantitative $/ \varepsilon /: / \varepsilon: /$ & be्lle /bعl/ bêele /bc:l/ \\
\hline \multirow[t]{3}{*}{ Ouverture et relâchement des voyelles /i, y, u/ } & vive [viv] [viv] \\
\hline & juste [3Yst] [3yst] \\
\hline & couve $[\mathrm{kvv}] \sim[\mathrm{kuv}]$ \\
\hline $\begin{array}{l}\text { Dévoisement et effacement des voyelles /i, y, u/ entre consonnes } \\
\text { sourdes }\end{array}$ & coutume $[$ kutym] $~[k: t y m]$ \\
\hline
\end{tabular}


informations délicates) en même temps qu'ils acceptent la publication des lectures du texte et des listes de mots (cf. annexe 11). En fin de compte, les 60 informateurs ont donné leur accord à la publication des enregistrements du texte lu et des listes de mots lues, alors que 12 des 60 informateurs ont refusé la publication des entretiens guidés. Par conséquent et pour des raisons d'uniformité du corpus, seuls les enregistrements de parole lue (lectures du texte et des listes de mots) seront mis à disposition de la communauté scientifique en open access.

Ainsi, un corpus d'une durée totale de 33 heures et 12 minutes a été mis en place - entretiens guidés, texte lu et listes de mots inclus (cf. tableau 17). Pour des raisons de comparabilité des données, les entretiens guidés n'ont pas été pris en compte dans les analyses des productions de locuteurs-modèles : l'analyse de formants des voyelles rendait en effet nécessaire la prise en compte de contextes phonétiques identiques pour en permettre la comparabilité. C'est la raison pour laquelle le choix s'est porté uniquement sur des mots (tirés des deux listes de mots) et des syntagmes (tirés du texte) lus, identiques pour tous les locuteurs, les entretiens guidés ayant uniquement été utilisés pour étayer qualitativement les données quantitatives récoltées à l’aide des questionnaires écrits :

Tableau 17 : Étendue du corpus selon les régions, le média et la situation de communication.

\begin{tabular}{|c|c|c|c|c|c|c|}
\hline \multirow[t]{2}{*}{ Région } & \multirow[t]{2}{*}{ Média } & \multicolumn{4}{|c|}{ Tâche } & \multirow[t]{2}{*}{ Total } \\
\hline & & $\begin{array}{l}\text { Entretiens } \\
\text { guidés }\end{array}$ & Texte lu & $\begin{array}{l}\text { Liste de } \\
\text { mots PFC }\end{array}$ & $\begin{array}{l}\text { Liste de } \\
\text { mots } 2\end{array}$ & \\
\hline \multirow[t]{2}{*}{ Paris } & Radio & 4h 14'44”' & $28^{\prime} 31^{\prime \prime}$ & $31^{\prime} 47^{\prime \prime}$ & $15^{\prime} 22^{\prime \prime}$ & 5h 30' 24"' \\
\hline & Télévision & 3 h 25'34"' & $28 ' 22 "$ & $34 ' 16 "$ & $16^{\prime} 10^{\prime \prime}$ & 4h 44' 22"' \\
\hline \multirow{2}{*}{$\begin{array}{l}\text { Suisse } \\
\text { romande }\end{array}$} & Radio & 4h 44'03'” & $26^{\prime} 25^{\prime \prime}$ & 33' 49' & 17'02'” & 6h 01'19" \\
\hline & Télévision & 5h 11'09" & 27 '29”' & 32’ 55”' & $16^{\prime} 14^{\prime \prime}$ & $6 \mathrm{~h} \mathrm{27’} 47^{\prime \prime}$ \\
\hline \multirow[t]{2}{*}{ Québec } & Radio & 4h 17'39”' & $28^{\prime} 21^{\prime \prime}$ & $37^{\prime} 41^{\prime \prime}$ & $18^{\prime} 33^{\prime \prime}$ & $5 h$ 42' $14 "$ \\
\hline & Télévision & 3h 27'00"' & $28^{\prime} 20^{\prime \prime}$ & $34 ' 12^{\prime \prime}$ & $16^{\prime} 25^{\prime \prime}$ & 4h 45' 57' \\
\hline Corpus & & 25h 20’09”' & 2h 47' 28’' & 3h 24' 40”' & 1h 39' 46"' & 33h 12'03" \\
\hline
\end{tabular}

Quant aux conditions des enregistrements, étant donné que les voyelles orales ont été soumises à une analyse de formants, j'ai veillé à l'obtention d'une très bonne qualité du signal sonore. Une grande partie des enregistrements a ainsi été effectuée soit dans une chambre sourde des stations de radio et des chaînes de télévision (cf. tableaux 12, 13 et 14) soit dans le bureau personnel des journalistesprésentateurs. Dans quelques cas (pour les locuteurs fpRom1, fpRff1, fpTjc1, fpTaf1, sgTmp1, sgTmm1, cqRgl1), le manque de locaux à disposition m’a obligé à faire les enregistrements dans l'une des salles communes de ces chaînes, raison pour laquelle des voix et de légers bruits de fond sont parfois audibles. Tous les 
enregistrements ont été effectués à l'aide d'un enregistreur ZOOM H4n au format .wav (44,1 KHz, stéréo, 16-bit) et ont été, par la suite, convertis en mono et normalisés (amplitude maximale à -1dB) à l'aide du logiciel Audacity.

\subsubsection{Protocole d'analyse}

L’analyse des voyelles produites par les locuteurs-modèles a été effectuée sur la base de syntagmes tirés du texte lu PFC et de mots tirés des deux listes de mots. Dans ce sous-chapitre, je présenterai tout d'abord les unités syntagmatiques et lexicales prises en compte lors des analyses (cf. 3.4.2.1) et j'aborderai par la suite les protocoles d'analyse des voyelles orales (cf. 3.4.2.2) et nasales (cf. 3.4.2.3).

\subsubsection{Choix des syntagmes et des mots à analyser}

Les traits de prononciation présentés dans les chapitres 2.3.2, 2.3.3 et 2.3.4 ont, dans un premier temps, été analysés dans un contexte à l'intérieur de syntagmes tirés des enregistrements du texte PFC.

\section{Syntagmes : Paris}

Dans le cas de Paris, les traits de prononciation parisiens à tester se trouvent ainsi dans les syntagmes présentés dans la colonne de droite du tableau 18 :

Tableau 18 : Syntagmes du texte du programme PFC contenant les traits parisiens à analyser dans la production des locuteurs-modèles.

\section{Traits parisiens à tester Syntagmes contenant ces traits}

1. Maintien de l'opposition /a/:/a/

- Syntagme : Et plus récemment, son usine de pâtes italiennes ( s'il faut montrer patte blanche pour circuler)

- Prononciation : [pat] [pat]

2. Maintien de l'opposition /e/ : / $\varepsilon$ / Tendance croissante à la loi de position :

- Syntagme (1) : Le Maire de Beaulieu est en [e] en syllabe finale ouverte, $[\varepsilon]$ en revanche très inquiet ( et plus récemment son usine de pâtes italiennes) syllabe finale fermée

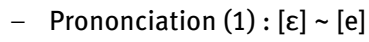

- Syntagme (2) : Jusqu'ici les seuls titres de gloire de Beaulieu étaient son vin blanc sec

- Prononciation (2) : [et $\varepsilon] \sim$ [ete] 
Tableau 18 (suite)

\section{Traits parisiens à tester \\ Syntagmes contenant ces traits}

\begin{tabular}{|c|c|c|}
\hline 3. & $\begin{array}{l}\text { Neutralisation de l'opposition } / ø /: / œ / \\
\text { Tendance croissante à la loi de position : } \\
\text { [ø] en syllabe finale ouverte, [œ] en } \\
\text { syllabe finale fermée }\end{array}$ & $\begin{array}{l}\text { - Syntagme : Quelques fanatiques auraient } \\
\text { même entamé un jeûne prolongé dans } \\
\text { l'église de Saint Martinville ( un jeune } \\
\text { membre de l'opposition aurait déclaré) } \\
\text { - Prononciation : [zœn] [3øn] }\end{array}$ \\
\hline 4. & 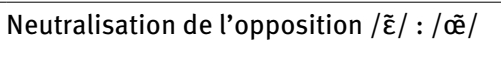 & 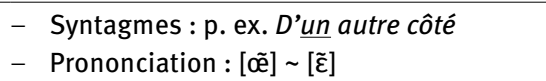 \\
\hline
\end{tabular}

Pour l'opposition / $\tilde{e} /: / \tilde{\varepsilon} /$, non pas un seul contexte, mais tous les contextes possibles du texte PFC (c'est-à-dire lors de l'apparition de <un> dans la graphie) ont été pris en compte. Il s'agit de six contextes pour l'article indéfini un. L’analyse des voyelles nasales reposant non pas sur des mesures de formants, mais sur des codages auditifs (cf. 3.4.2.3), j'ai préféré prendre en compte le plus de codages possible pour permettre une plus grande fiabilité de ces codages.

Soulignons par ailleurs que pour la paire de voyelles $[\varepsilon] \sim[e]$, deux syntagmes ont été pris en compte, avec dans un cas la forme est (opposée à la conjonction et extraite d'un autre syntagme) et dans l'autre, la forme étaient. Ce choix repose sur le fait que l'état actuel de la recherche observe une différence entre ces deux formes : alors qu'une tendance à la fermeture en [e] est observée pour la forme est (cf. Hansen/Juillard 2011, 315), la paire minimale été /ete/ étai(en)t /ete/ est encore souvent attestée dans plusieurs études (cf. p. ex. Lyche/Østby 2009, 212 ; Hansen 2017, 128-129; chapitre 1.3.2).

Mentionnons finalement que, faute d'extraits appropriés dans le texte et les listes de mots, deux des traits parisiens mentionnés dans le tableau 6 (cf. chapitre 2.3.2) n'ont pas été pris en compte dans l'analyse des productions et ne figurent donc pas dans le tableau 18. Il s'agit du maintien de l'opposition /o/ : / / (p. ex. paume /pom/ pomme /pom/) ainsi que de la postériorisation en chaîne des

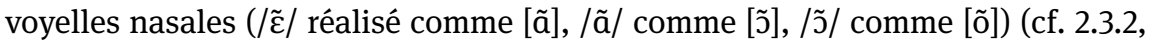
tableau 6).

\section{Syntagmes : Suisse romande}

Dans le cas de la Suisse, les traits de prononciation ayant fait l'objet des analyses des productions se trouvent dans les syntagmes présentés dans la colonne de droite du tableau 19. 
Tableau 19 : Syntagmes du texte du programme PFC contenant les traits suisses romands à analyser dans la production des locuteurs-modèles.

\begin{tabular}{|c|c|c|}
\hline & Traits suisses romands à tester & Syntagmes contenant ces traits \\
\hline 1. & $\begin{array}{l}\text { Opposition quantitative en syllabe } \\
\text { finale fermée (+ différence de timbre) }\end{array}$ & $\begin{array}{l}\text { - Syntagme : Et plus récemment, son usine } \\
\text { de pâtes italiennes ( s'il faut montrer patte } \\
\text { blanche pour circuler) } \\
\text { - Prononciation : } \\
\text { [pat] } \sim \text { [pa:t] [pa:t] }\end{array}$ \\
\hline 2. & $\begin{array}{l}\text { Opposition quantitative en syllabe } \\
\text { finale ouverte (+ diphtongaison) }\end{array}$ & $\begin{array}{l}\text { - } \text { Syntagme : En fin d'année } \\
\text { - Prononciation : } \\
\quad \text { [ane] } \sim \text { [ane:] } \sim \text { [ane:'] }\end{array}$ \\
\hline 3. & Opposition / $\tilde{\varepsilon} /:$ / ̃̃/ & $\begin{array}{l}\text { - Syntagme : p. ex. D’un autre côté } \\
\text { - Prononciation : }[\tilde{e}] \sim[\tilde{\varepsilon}]\end{array}$ \\
\hline 4. & Préférence de la diérèse à la synérèse & 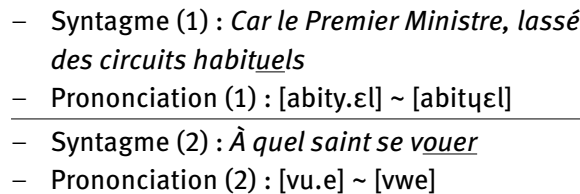 \\
\hline
\end{tabular}

Notons que, pour les mêmes raisons que dans le cas de Paris (cf. supra), l'analyse de l'opposition / $\tilde{\varepsilon} /$ : /õ̃/ repose non pas sur un seul contexte, mais sur tous les contextes possibles du texte PFC ( 6 contextes pour l'article indéfini un).

Par ailleurs, deux syntagmes ont été pris en compte dans le cas de la différence entre les diérèses et les synérèses, afin d'obtenir un contexte pour au moins deux des paires glissante-voyelle possibles $([\mathrm{i}] \sim[\mathrm{j}],[\mathrm{y}] \sim[\mathrm{u}],[\mathrm{u}] \sim[\mathrm{w}]):$ le mot habituels contient la paire $[\mathrm{y}] \sim[\mathrm{u}]$ et le mot vouer la paire $[\mathrm{u}] \sim[\mathrm{w}]$. Aucun contexte n’a cependant pu être trouvé pour la paire [i] [j].

Soulignons finalement qu'aucun extrait n'a pu être trouvé pour l'opposition /e/ : / / / en syllabe finale ouverte (p. ex. je pourrai /e/ je pourrais / / /) (cf. 2.3.3, tableau 7). Ce trait n'a donc pas pu être testé dans un contexte syntagmatique.

\section{Syntagmes : Québec}

Pour finir, les traits de prononciation québécois soumis aux analyses de production se trouvent dans les syntagmes présentés dans la colonne de droite du tableau 20.

Notons ici aussi que, comme l'analyse des voyelles nasales repose sur des codages auditifs (cf. 3.4.2.3), toutes les occurrences possibles présentes dans le texte PFC ont été prises en compte (6 contextes pour l'opposition / $\tilde{\varepsilon} /$ : /õe/, 19 contextes pour la réalisation $\mathrm{du} / \tilde{\varepsilon} /$ et 50 contextes pour la réalisation $\mathrm{du} / \tilde{a} /$ ). 
Tableau 20 : Syntagmes du texte du programme PFC contenant les traits québécois à analyser dans la production des locuteurs-modèles.

\begin{tabular}{|c|c|c|}
\hline & Traits québécois à tester & Syntagmes contenant ces traits \\
\hline 1. & Opposition /a/: /a/ & $\begin{array}{l}\text { - Syntagme : Et plus récemment, son usine de pâtes } \\
\text { italiennes }(\sim \text { s'il faut montrer patte blanche pour } \\
\text { circuler }) \\
\text { - Prononciation }:[\mathrm{pat}] \sim[\mathrm{pat}]\end{array}$ \\
\hline 2. & $\begin{array}{l}\text { /a/ final : [a] ou [a] (variation } \\
\text { selon le mot) } \\
\text { /a/ final dans <-oi> : [wa] ou [wa] } \\
\text { (variation selon le mot et le type } \\
\text { de syllabe) }\end{array}$ & $\begin{array}{l}\text { - Syntagme : Trois échevins } \\
\text { - Prononciation : [tвwa] [tвwa] }\end{array}$ \\
\hline 3. & Opposition / $\tilde{\varepsilon} /: / \tilde{e} /$ & $\begin{array}{l}\text { - Syntagme : p. ex. D’un autre côté } \\
\text { - Prononciation : }[\propto \tilde{e}] \sim[\tilde{\varepsilon}]\end{array}$ \\
\hline 4. & Réalisation de / $\tilde{\varepsilon} /$ comme [ẽ] & $\begin{array}{l}\text { - Syntagme : p. ex. Dans le coin } \\
\text { - Prononciation : }[k w \tilde{\varepsilon}] \sim[k w \tilde{e}]\end{array}$ \\
\hline 5. & 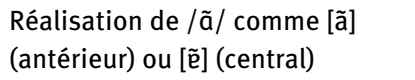 & $\begin{array}{l}\text { - Syntagme : p. ex. L'ancienne poste vendue } \\
\text { - Prononciation : [vãdy] } \sim[\text { vẽdy] } \sim \text { [vãdy] }\end{array}$ \\
\hline 6. & $\begin{array}{l}\text { Ouverture et relâchement des } \\
\text { voyelles } / \mathrm{i}, \mathrm{y}, \mathrm{u} / \text { en finale de mot } \\
\text { se terminant par une ou plusieurs } \\
\text { consonne(s) non allongeante(s) }\end{array}$ & $\begin{array}{l}\text { - Syntagme : Autour des mêmes villes } \\
\text { - Prononciation : [vil] [vil] }\end{array}$ \\
\hline 7. & $\begin{array}{l}\text { Dévoisement (ou effacement) } \\
\text { des voyelles } \\
\text { /i, y, u/ entre consonnes sourdes }\end{array}$ & 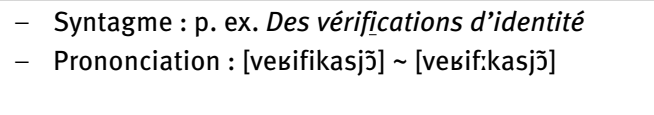 \\
\hline
\end{tabular}

Mentionnons par ailleurs que l'analyse du dévoisement (ou effacement) des voyelles / $\mathrm{i}, \mathrm{y}, \mathrm{u}$ / se différencie également de celles des autres traits vocaliques en ce sens qu'elle a été effectuée sur la base d'une lecture qualitative de spectrogramme et d'un système de codage ternaire (cf. 3.4.2.2.2). Étant donné ce codage, le choix s'est à nouveau porté sur l'analyse de ce trait non pas dans un seul lexème, mais dans tous les contextes potentiels pouvant être relevés pour le /i/, en sous-entendant que les dévoisements et effacements potentiels sont sans doute similaires pour les voyelles /i/, /y/ et /u/. Dans le cas des listes de mots, il s'agit des lexèmes piquet, piqqué, piquais, piquer (dans lesquels le /i/ s'avère cependant se trouver dans un contexte phonologique identique, ce qui n'est pas le cas des contextes trouvés dans le texte PFC ; cf. infra).

Par ailleurs, faute d'extraits correspondants, ni l'opposition quantitative $/ \varepsilon /$ vs. $/ \varepsilon: /(p$. ex. faites $/ \varepsilon / \sim$ fête $/ \varepsilon: /)$ ni les diphtongaisons des voyelles allongées (p. ex. neige $\left.\left[\mathrm{na}^{\varepsilon_{3}}\right] \sim\left[\mathrm{na}^{\mathrm{e}} 3\right] \sim\left[\mathrm{na}^{\mathrm{i}} 3\right]\right)$ n'ont pu être analysées dans un contexte syntagmatique (cf. 2.3.4, tableau 8). 


\section{Mots isolés : Paris}

Dans un deuxième temps, après avoir effectué les analyses des traits de prononciation en contexte syntagmatique, ces mêmes traits ont été analysés dans des contextes à l'intérieur de mots isolés, ces mots ayant été tirés soit de la liste de mots PFC, soit de la liste de mots complémentaire (cf. colonne de droite dans chaque tableau). Notons que dans les cas où des paires minimales ont été prises en compte (patte pâte, épais $\sim$ épée, jeune jeûne, brin brun), les mots n’apparaissaient pas directement les uns après les autres (comme c'est le cas des 10 derniers mots de la liste de mots PFC ; cf. annexe 8), mais à différents endroits dans la liste. Dans le cas de Paris, le choix s'est donc porté sur les mots suivants :

Tableau 21 : Mots tirés de la liste de mots PFC contenant les traits parisiens à analyser dans la production des locuteurs-modèles.

\begin{tabular}{|c|c|c|c|}
\hline & Traits parisiens à tester & Mots contenant ces traits & Liste de mots \\
\hline 1. & Maintien de l'opposition /a/ : /a/ & patte /pat/ pâate /pat/ & Liste PFC \\
\hline 2. & $\begin{array}{l}\text { Maintien de l'opposition } / \mathrm{e} /: / \varepsilon / \\
\text { Tendance croissante à la loi de position : } \\
\text { [e] en syllabe finale ouverte, }[\varepsilon] \text { en } \\
\text { syllabe finale fermée }\end{array}$ & $\begin{array}{l}\text { épée /epe/ } \\
\text { épais /epe/ /epe/ }\end{array}$ & Liste PFC \\
\hline
\end{tabular}

3. Neutralisation de l'opposition /ø/:/œ/ jeune/zœn/ jeûne /ろøn/ Liste PFC Tendance croissante à la loi de position :

[ø] en syllabe finale ouverte, [œ] en syllabe finale fermée

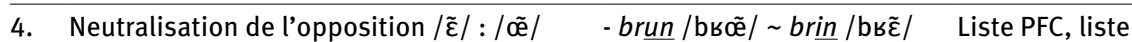

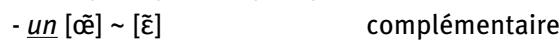

Concernant l'opposition /e/ : / $\varepsilon$, notons que la paire de mots utilisée pour l'analyse de mots isolés (épée épais) ne correspond pas aux mots se trouvant dans les syntagmes analysés (Le village de Beaulieu est en grand émoi ; Jusqu'ici les seuls titres de gloire de Beaulieu étaient son vin blanc sec). Ainsi, étant donné que les paires minimales été $~ e ́ t a i(e n) t$ et épée $\sim$ épais sont encore attestées dans plusieurs études alors que l'on observe une tendance actuelle à la fermeture en [e] de la forme est, il est probable que les analyses acoustiques révèlent des valeurs différentes pour les voyelles produites dans épée épais et étaient d'une part, et dans est d'autre part.

Par ailleurs, deux commentaires s'imposent par rapport à l'opposition / $\tilde{\varepsilon} /$ : /õ̃/ : premièrement, de manière similaire au cas des syntagmes (cf. supra), je n'ai 
pas pris en compte un contexte unique dans les codages auditifs (cf. 3.4.2.3), mais tous les contextes possibles, c'est-à-dire tous ceux contenant un <un> graphique. Il s'agit dans les deux listes de mots de l'article indéfini un (9 contextes) et de l'adjectif brun (2 contextes). Deuxièmement, les interprétations devront prendre en compte le fait que les deux lexèmes apparaissant dans ces listes de mots (brun et un) peuvent engendrer différents résultats du point de vue du comportement de /œẽ/. Il semble en effet exister un conditionnement lexical et prosodique favorisant une disparition du [œ̃] plus rapide dans certains contextes (cf. Hansen 1998, 106-107). Walter (1976, 319-338), tout d’abord, évoque la fréquence pour expliquer la disparition plus rapide observable dans certains mots par rapport à d'autres : des mots plus rares comme jungle, bungalow ou tungstène auraient, selon elle, tendance à garder plus longtemps le timbre [õ]] que des mots très fréquents comme un, lundi ou brun. Les observations de Nève de Mévergnies $(1984,211)$ confirment ces hypothèses et y ajoutent le facteur de l'accentuation : la confusion entre / $\tilde{\varepsilon} /$ et /œẽ/ débuterait par l'article enclitique un inaccentué et le mot lundi (en raison d'une harmonie vocalique avec le /i/ final). Elle se poursuivrait par le un numéral et les dérivés de un (quelqu'un, chacun, aucun) ainsi que, par la suite, par les autres mots contenant un /œẽ/ inaccentué. Finalement, elle se terminerait par les mots contenant un /õ̃/ accentué. Ainsi, pour l'analyse du présent corpus, il est probable que chez certains locuteurs, un [œẽ] soit observable dans brun alors qu'il ne l'est pas dans un.

Soulignons finalement que le maintien de l'opposition /o/ : / / (p. ex. paume /pom/ pomme /pom/) (cf. 2.3.2, tableau 6) et la postériorisation en chaîne des

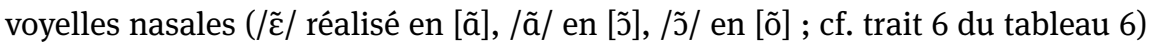
n’ont pas non plus été pris en compte ici.

\section{Mots isolés : Suisse romande}

Dans le cas de la Suisse romande, les traits de prononciation présentés dans le tableau 7 (cf. chapitre 2.3.3) ont été analysés dans le contexte des mots se trouvant dans le tableau 22. Notons que, comme dans le cas de Paris, l'analyse de paires minimales (patte pâte, penser pensée, pourrai pourrais, jeune $\sim$ jeûne, brin brun) porte sur des mots n'apparaissant pas directement les uns après les autres mais bien à différents endroits dans la liste (cf. annexe 8).

Ici aussi, l'analyse de l'opposition / $\tilde{\varepsilon} /:$ / ẽ/ repose sur tous les contextes contenant un <un> graphique (un : 11 contextes; brun : 2 contextes). Ainsi, il est important - comme dans le cas de Paris (cf. supra) - de prendre en compte la probable différence de prononciation de $<$ un $>$ dans brun et dans l'article indéfini un. Cependant, selon Andreassen/Maître/Racine (2010), il semble que dans ce cas-ci le un soit, au contraire de Paris, plus résistant au processus de neutralisation, 
Tableau 22 : Mots tirés des deux listes (liste PFC et liste complémentaire) contenant les traits suisses romands à analyser dans la production des locuteurs-modèles.

\begin{tabular}{|c|c|c|c|}
\hline & Traits suisses romands à tester & Mots contenant ces traits & Liste de mots \\
\hline 1. & $\begin{array}{l}\text { Opposition quantitative en syllabe } \\
\text { finale fermée (+ différence de timbre) }\end{array}$ & $\begin{array}{l}\text { patte /pat/ } \\
\text { pâte /pa:t/ /pa:t/ }\end{array}$ & Liste PFC \\
\hline 2. & $\begin{array}{l}\text { Opposition quantitative en syllabe } \\
\text { finale ouverte (+ diphtongaison) }\end{array}$ & $\begin{array}{l}\text { penser /pãse/ } \\
\text { pensée /pãse:/ /pãse:j/ }\end{array}$ & Liste complémentaire \\
\hline 3. & $\begin{array}{l}\text { Opposition } / \mathrm{e} /: / \varepsilon / \text { en syllabe finale } \\
\text { ouverte (distinction de certains } \\
\text { temps et modes) }\end{array}$ & $\begin{array}{l}\text { je pourrai /puве/ } \\
\text { je pourrais /puвє/ }\end{array}$ & Liste complémentaire \\
\hline 4. & Opposition / $\tilde{\varepsilon} /:$ / ̃̃/ & 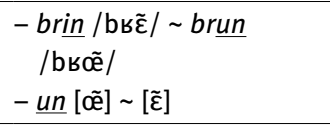 & $\begin{array}{l}\text { Liste PFC, liste } \\
\text { complémentaire }\end{array}$ \\
\hline 5. & $\begin{array}{l}\text { Préférence de la diérèse à la } \\
\text { synérèse }\end{array}$ & 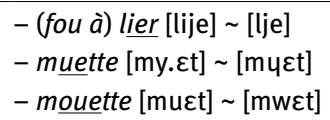 & Liste PFC \\
\hline
\end{tabular}

« [...] le timbre arrondi étant produit par des locuteurs qui ne connaissent que / $\tilde{\varepsilon} /$ dans la paire minimale brin-brun »(Andreassen/Maître/Racine 2010, 224).

Dans le cas des diérèses et synérèses, de manière similaire aux analyses effectuées dans le cadre du texte PFC, un contexte a été pris en compte pour chacune des paires glissante-voyelle $([\mathrm{i}] \sim[\mathrm{j}],[\mathrm{y}] \sim[\mathrm{u}],[\mathrm{u}] \sim[\mathrm{w}])$ : (fou à) lier ([lije] [lje]), muette $([\mathrm{my \varepsilon t}] \sim[\mathrm{mu \varepsilon t}])$ et mouette $([\mathrm{mu \varepsilon t}] \sim[\mathrm{mw \varepsilon t}])$.

\section{Mots isolés : Québec}

Pour finir, les traits de prononciation québécois se trouvant dans le tableau 8 ont été soumis à des analyses dans le contexte des mots se trouvant dans le Tableau 23. Soulignons à nouveau que les mots touchés par l'analyse de paires minimales (patte pâte, faites fête, brin brun) apparaissent à différents endroits dans la liste et non directement les uns après les autres (cf. annexe 8).

Notons que, dans le cas du Québec également, les voyelles nasales ont été analysées non pas sur la base d'un seul contexte, mais de tous les contextes possibles dans les deux listes de mots. Il s'agit des mots un ( 7 contextes) et brun ( 2 contextes) dans le cas de l'opposition / $\tilde{\varepsilon} /:$ /õe/, de 68 contextes contenant les graphies <in>, $<\mathrm{im}>,\langle\mathrm{ym}>$ et certains $<$ en $>$ dans le cas de la réalisation du $/ \tilde{\varepsilon} /$ et de 81 contextes contenant les graphies <an> et <en> dans le cas de la réalisation du /ã/.

Mentionnons par ailleurs à propos de l'opposition /õ̃/: / $\tilde{\varepsilon} /$ que le problème du comportement potentiellement différent du /õ/ dans brun et un se pose également dans le cas du Québec. Il est cependant probable que les résultats s'op- 
Tableau 23 : Mots tirés des deux listes (liste PFC et liste complémentaire) contenant les traits québécois à analyser dans la production des locuteurs-modèles.

\begin{tabular}{|c|c|c|c|}
\hline & Traits québécois à tester & Mots contenant ces traits & Liste de mots \\
\hline 1. & Opposition /a/ : /a/ & patte /pat/ pâate /pat/ & Liste PFC \\
\hline 2. & $\begin{array}{l}-\quad \text { /a/ final : [a] ou [a] } \\
\quad \text { (variation selon le mot) } \\
\text { - } \quad \text { /a/ final dans <-oi>: [wa] ou } \\
\text { [wa] (variation selon le mot } \\
\text { et le type de syllabe) }\end{array}$ & 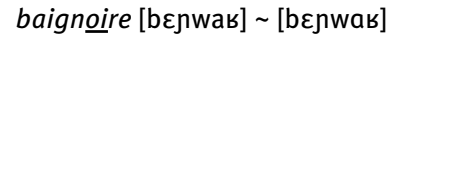 & Liste PFC \\
\hline 3. & $\begin{array}{l}\text { Opposition quantitative } / \varepsilon / \\
\text { vs. / } \varepsilon \text { :/ }\end{array}$ & faites $/ \varepsilon / \sim$ fête $/ \varepsilon: /$ & Liste PFC \\
\hline 4. & $\begin{array}{l}\text { Diphtongaisons des voyelles } \\
\text { allongées }\end{array}$ & fête $[\mathrm{f} \varepsilon: \mathrm{t}] \sim\left[\mathrm{fa}^{3} \mathrm{t}\right]$ & Liste PFC \\
\hline 5. & Opposition / $\tilde{\varepsilon} /: / \tilde{e} /$ & 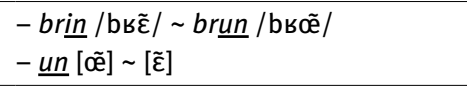 & $\begin{array}{l}\text { Liste PFC, liste } \\
\text { complémentaire }\end{array}$ \\
\hline 6. & Réalisation de / $\tilde{\varepsilon} /$ en [ẽ] & p. ex. médecin [meds $\tilde{\varepsilon}] \sim$ [medsẽ] & $\begin{array}{l}\text { Liste PFC, liste } \\
\text { complémentaire }\end{array}$ \\
\hline 7. & $\begin{array}{l}\text { Réalisation de /ã / en [ã] } \\
\text { (antérieur) ou [ẽ] (central) }\end{array}$ & p. ex. penser [pãse] [pẽse] [pãse] & $\begin{array}{l}\text { Liste PFC, liste } \\
\text { complémentaire }\end{array}$ \\
\hline 8. & $\begin{array}{l}\text { Ouverture et relâchement des } \\
\text { voyelles /i, y, u/ en finale de } \\
\text { mot se terminant par une ou } \\
\text { plusieurs consonne(s) non } \\
\text { allongeante(s) }\end{array}$ & $\operatorname{six}[\mathrm{sis}] \sim[\mathrm{sIs}]$ & Liste PFC \\
\hline 9. & $\begin{array}{l}\text { Dévoisement (ou effacement) } \\
\text { des voyelles /i, y, u/ entre } \\
\text { consonnes sourdes }\end{array}$ & p. ex. piquer [pike] [p:ke] & Liste PFC \\
\hline
\end{tabular}

posent à ceux de Paris et de la Suisse romande, étant donné que le maintien de l'opposition /õ̃/ : / $/ \tilde{\varepsilon} /$ est unanimement considéré comme très stable au Québec (cf. 2.3.4), seul l'article défini un pouvant dans quelques rares cas présenter la prononciation [ $\tilde{\varepsilon}$ ] (cf. Léon 1983, 55). La variation lexicale pourrait par ailleurs également jouer un rôle dans l'analyse de la réalisation $\mathrm{du} / \mathrm{wa}$ / dans baignoire et trois, l'état actuel de la recherche considérant le [wa:] dans la syllabe finale fermée de baignoire et le [wa] dans la syllabe finale ouverte de trois comme étant les plus "standards » (cf. 2.3.4, tableau 9). Mentionnons en outre que la réalisation de / $\tilde{\varepsilon} /$ dans médecin coin, celle de /ã/dans penser vendue ainsi que celle de /i/ dans $s \underline{i} x \sim$ ville pourraient également être sujettes à l'influence de la variation lexicale.

Des précisions doivent également être apportées par rapport à l'analyse du dévoisement et de l'effacement des voyelles /i, y, u/ dans ce texte PFC : en effet, comme dans le cas des mots isolés (cf. supra), cette analyse a été effectuée sur un 
nombre maximal d'occurrences $\mathrm{du} / \mathrm{i} /$ entre consonnes sourdes. Ce faisant, huit lexèmes ont pu être relevés (jusqu'îci, officielles, vérifications, identité, situation, participation, particulier), permettant ainsi une analyse plus détaillée des effets lexicaux.

Comme déjà mentionné brièvement dans le présent sous-chapitre, les analyses des voyelles orales et nasales figurant dans les tableaux 21, 22 et 23 ont été effectuées de deux manières fondamentalement différentes. En effet, en raison des différents effets acoustiques de la nasalisation, comme les transitions des formants, l'augmentation de la largeur des bandes de formants et la présence d'antiformants dus aux antirésonances dans le signal acoustique (cf. Delvaux 1999, 8 ; cf. également Delvaux 2006 ; Carignan 2011), une analyse acoustique de voyelles nasales est particulièrement difficile et peu fiable sans avoir également des données sur l'articulation (cf. Carignan 2012, 747). Par conséquent, alors que les voyelles orales ont fait l'objet d'analyses acoustiques (analyses de formants dans le cas d'oppositions de timbre, analyses de durées vocaliques dans le cas d'oppositions de durées ; cf. 3.4.2.2), les voyelles nasales ont été codées auditivement par accord interjuges (cf. 3.4.2.3). C'est la raison pour laquelle les protocoles de ces deux types d'analyse seront présentés séparément ci-dessous.

\subsubsection{Analyses acoustiques des voyelles orales}

\subsection{Segmentation}

Dans un premier temps, les syntagmes et mots contenant les voyelles orales à analyser (cf. 3.4.2.1) ont été segmentés manuellement à l'aide du logiciel PRAAT (cf. Boersma/Weenink 2016). Ce faisant, les frontières du début et de la fin de chacune des voyelles ont été placées sur la base des critères standards définis par Schwab et al. (1998, 2-3) : dans la plupart des cas, la segmentation a été effectuée en fonction des formants $\mathrm{F}_{2}$ et $\mathrm{F}_{3}$. Dans les cas de voyelles voisines présentant des structures formantiques trop peu distinctes (comme [y] et $[\varepsilon]$ dans habituels et muette, cf. tableaux 19 et 22), la différenciation s'est faite sur la base d'un changement observable au niveau de la fréquence fondamentale et/ou de l'amplitude globale du signal (cf. figure 7).

Notons que la mention de ces conventions de segmentation, qui ont été appliquées de manière systématique à toutes les voyelles segmentées, est importante, étant donné que le recours à un autre type de segmentation aurait pu aboutir à des résultats différents.

Les voyelles ainsi segmentées, des mesures ont été effectuées sur les voyelles, ces mesures se divisant en deux catégories distinctes : toutes les oppositions de timbre ont été analysées sur la base d'analyses de formants (cf. 3.4.2.2.2), alors 


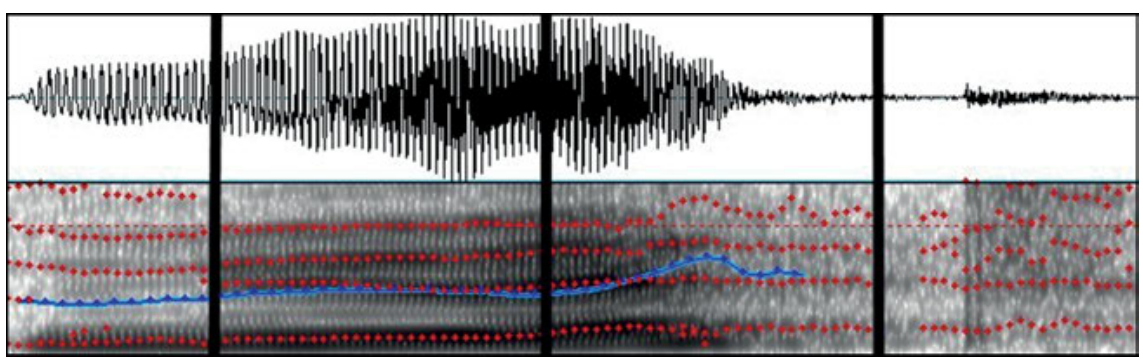

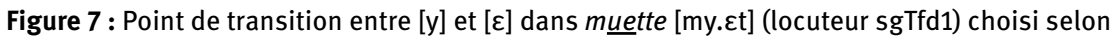
un changement dans la fréquence fondamentale et dans l'amplitude globale.

que les oppositions quantitatives ont été analysées sur la base des durées vocaliques (cf. 3.4.2.2.3).

\subsection{Mesures de formants}

Les analyses de formants ont été effectuées sur la base de la fréquence des deux premiers formants $\left(\mathrm{F}_{1}, \mathrm{~F}_{2}\right)$, le troisième formant, associé à la labialité (cf. Vais-

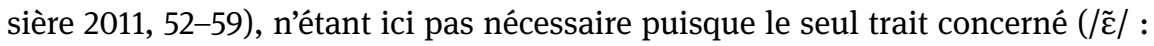
/œẽ/) est nasal et donc analysé, dans le présent ouvrage, sous forme de codages par accord interjuges (cf. 3.4.2.1). Notons cependant que les mesures sont différentes selon le type de voyelle.

Dans le cas (le plus fréquent) des voyelles non diphtonguées (p. ex. patte /pat/ pâte /pat/, épée /epe/ épais /epc/, jeune /3œn/ jê̂ne /3øn/), les formants ont été extraits sous PRAAT à l'aide d'un script les mesurant à 50\% de la durée vocalique. Ce faisant, le formant maximum (F5) a été placé à $5000 \mathrm{~Hz}$ pour les hommes et à $5500 \mathrm{~Hz}$ pour les femmes, avec une fenêtre temporelle (Gaussian) de 0,025 s. La signification statistique des différences observées entre les fréquences formantiques des voyelles produites par les locuteurs-modèles a ensuite été vérifiée, à l'aide du logiciel libre $R$, sur la base du test de Wilcoxon-MannWhitney, un test non paramétrique permettant de comparer deux échantillons indépendants de petite taille, le seuil de signification statistique ayant été établi à 0,05 (cf. Riverin-Coutlée 2013, 91-92).

Dans le cas des voyelles diphtonguées (cf. trait 4 du tableau 23 : fête [ع:] $\left[\mathrm{a}^{3}\right]$ ), le même script $P R A A T$ a été adapté pour extraire les formants à $25 \%$ et $75 \%$ de chaque voyelle, afin de pouvoir retracer le déplacement des formants lorsque le $/ \varepsilon:$ / est diphtongué. Le même test de Wilcoxon-Mann-Whitney a ensuite été appliqué aux résultats sous $R$ afin d'en vérifier la signification statistique.

Notons pour ces deux premiers cas que jusqu'ici, différentes procédures de normalisation ont été proposées dans le but de prendre en compte la variation 
des fréquences observées due aux caractéristiques physiologiques des locuteurs (cf. Nearey 1989 ; Syrdal/Gopal 1986). Ces procédures présentent cependant des inconvénients (cf. Disner 1980 ; Adank 2003), notamment la tendance à masquer la variation intra-individuelle. Par ailleurs, étant donné que (1) le corpus sur lequel reposent les mesures, avec ses 20 locuteurs par région, est assez vaste pour que les mesures puissent être présentées en hertz sans avoir recours à une normalisation (cf. Woehrling 2009, 70), et que (2) l'objectif des mesures de formants n'est pas de reconstituer un espace vocalique F1/F2 mais bien d'opposer ponctuellement les valeurs formantiques de voyelles se trouvant majoritairement dans des paires minimales pour y déceler une différence (ou non), la présentation des valeurs formantiques se fera sans recours à une normalisation intrinsèque ou extrinsèque ni à une échelle de type Bark.

Dans le cas des synérèses ou diérèses (cf. trait 4 du tableau 19 : habituels [abity.cl] [abitucl] ; trait $5 \mathrm{du}$ tableau 22 : fou à lier [lije] [lje], muette [my.ct] [muct], mouette [muct] [mwct]), la différence ne peut pas être mesurée de manière ponctuelle comme pour les voyelles (non) diphtonguées, mais seulement par l'analyse du segment entier. L'utilisation d'une diérèse est ainsi déterminée par l'apparition d'une voyelle (p. ex. [y]), alors que l'utilisation d'une synérèse est déterminée par l'apparition d'une semi-voyelle (p. ex. [ч]). Cette semi-voyelle est caractérisée par une structure formantique faible, instable et en variation constante. Ainsi, elle ne possède aucune partie stable et son caractère transitoire et vocalique rend impossible la segmentation entre la semi-voyelle et la voyelle qui

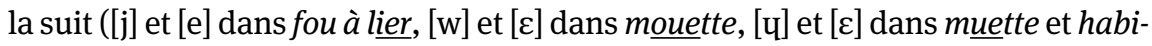
tuels) (cf. Meunier 2007, 169). Au contraire, la voyelle présente cette partie stable (cf. figures 8 et 9 ) :

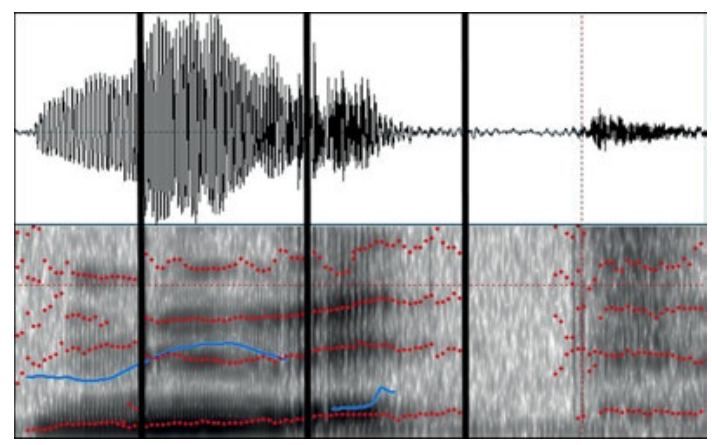

Figure 8 : Diérèse présentant deux voyelles distinctes $([y] /[\varepsilon])$ ayant chacune une partie stable. 


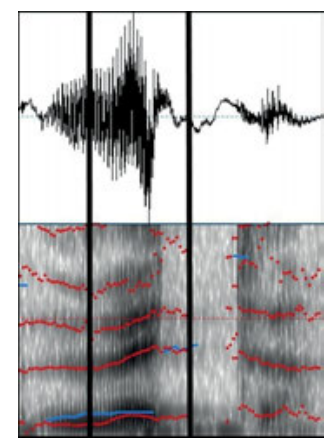

Figure 9 : Synérèse présentant une semi-voyelle [u] instable et en variation permanente (formants F2 et $\mathrm{F} 3$ ascendants) et ne pouvant être segmentée de la voyelle qui la suit $([\varepsilon])$.

Ainsi, les analyses des deux contextes de synérèses et diérèses (cf. trait $4 \mathrm{du}$ tableau 19 : habituels [abity.cl] [abitucl] ; trait 5 du tableau 22 : fou à lier [lije] [lje], muette [my.ct] [muct], mouette [muct] [mwst]) ont été effectuées sur la base d'une lecture qualitative de spectrogramme, dans laquelle les formants $F_{2}$ et $\mathrm{F}_{3}$ - également utilisés pour la segmentation (cf. 3.4.2.2.1) - ont été considérés quant à leur stabilité/leur variation. Ce faisant, chaque segment présentant une partie stable et pouvant être séparé clairement de la voyelle suivante a été considéré comme une voyelle ([i], [y] ou [u]), alors que tout segment instable ne pouvant être séparé de la voyelle suivante a été considéré comme semi-voyelle ([j], [u] ou [w]). Les résultats de ces lectures de spectrogramme ont ainsi été codés selon un codage binaire ( 1 = synérèse, 2 = diérèse) sous PRAAT. Par la suite, les données ont été extraites à l'aide de l'outil DOLMEN (cf. Eychenne/Paternostro 2016) et analysées statistiquement en utilisant le logiciel libre $R$. Ce faisant, j'ai opté pour la méthode du chi carré de Pearson, étant donné que les données d'un tel codage binaire sont structurées sous forme de proportions.

Quant à l'analyse du dévoisement (ou de l'effacement) de /i, y, u/ (cf. trait 7 du tableau 20 : des vérifications d'identité [veвifikasjõ] [vевif:kasjõ] ; trait $9 \mathrm{du}$ tableau 23 : piquer [pike] [p:ke]), elle a également été effectuée sur la base d'une lecture qualitative de spectrogramme, la présence ou l'absence d'un voisement ne pouvant pas non plus être mesurée de manière ponctuelle mais seulement à travers une analyse du segment entier. Ainsi, la différence entre les variantes voisées et dévoisées a été détectée sur la base de la présence ou de l'absence de la barre de voisement (dont la fréquence est celle de la fréquence fondamentale (F0) ; cf. Meunier 2007, 168), et celle entre une voyelle dévoisée et l'absence totale de voyelle sur la base de la présence ou de l'absence de formants vocaliques (cf. Jacques 1990, 60). Les résultats ont alors été codés selon un codage ternaire sous $P R A A T$, le code (1) correspondant à une voyelle voisée, le code (2) à une voyelle dévoisée et le code (3) à une voyelle effacée : 
1. Voyelle voisée : voyelle présentant une barre de voisement et des formants. Notons qu'une barre de voisement partielle, c'est-à-dire n'apparaissant pas sur toute la durée de la voyelle, a également mené à considérer la voyelle comme voisée (p. ex. université [ynivessite]).

2. Voyelle dévoisée : voyelle ne présentant pas de barre de voisement, mais des formants vocaliques (p. ex. université [ynivєssite]).

3. Syncope de la voyelle : aucune voyelle détectable en raison de l'absence de formants, mais allongement de la consonne précédente (p. ex. université [ynivess:te]).

L'extraction des données a, ici aussi, été effectuée à l'aide de l'outil DOLMEN (cf. Eychenne/Paternostro 2016) et les tests statistiques en recourant au logiciel libre $R$. Ce faisant, la signification des différences entre les fréquences statistiques des trois types de réalisation $\mathrm{du} / \mathrm{i} / \mathrm{a}$, ici aussi, été effectuée sur la base de la méthode du chi carré de Pearson.

\subsection{Mesures quantitatives des durées vocaliques}

Toutes les mesures des durées vocaliques ont été effectuées sous PRAAT en millisecondes (= ms) et les différences entre les résultats vérifiées quant à leur signification statistique sous $R$ à nouveau sur la base du test Wilcoxon-Mann-Whitney. Notons que toutes ces mesures ont été combinées à des mesures de formants présentées au chapitre précédent (cf. 3.4.2.2.2), étant donné que dans toutes les unités lexicales utilisées, la durée vocalique peut apparaitre soit avec une diphtongaison (faites /fct/ fête /fع:t/ /fa ${ }^{3} \mathrm{t} /$ au Québec, penser /pãse/ pensée /pãse:/ /pãse:j/ et année [ane] [ane:] [ane:'] en Suisse romande), soit avec une simple différence de timbre (patte /pat/ p pâte /pa:t/ /pa:t/ en Suisse romande).

\subsubsection{Analyse des voyelles nasales}

Afin d'éviter toute erreur de calcul de formants due aux antirésonances présentes dans le signal acoustique des voyelles nasales (cf. 3.4.2.2.1), ces voyelles ont été codées auditivement sur la base d'un système par accord interjuges. Le codage a été réalisé pour chacune des régions par deux étudiant·e.s de linguistique natifs/ natives d'une des variétés de la région en question et à l'aide du logiciel PRAAT. ${ }^{9}$ Notons que pour la Suisse romande, j'ai moi-même réalisé l'un des codages.

Les enregistrements des trois régions ont été analysés par rapport à la ques-

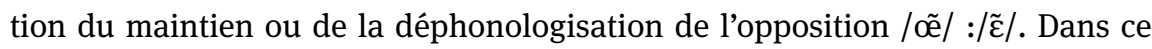

9 Les honoraires de ces étudiants ont été financés par l'Université de Vienne. 
cas-ci, le codage est binaire : les codeurs avaient donc la possibilité de coder les voyelles nasales entendues soit par un 1, un codage représentant une voyelle arrondie [œẽ], soit par un 2, un codage représentant une voyelle non arrondie [ $\tilde{\varepsilon}]$. Le codage a été effectué dans tous les contextes des deux listes de mots et du texte lu dans lesquels une voyelle nasale arrondie [œẽ] était susceptible d'apparaître, c'est-à-dire tous les contextes correspondant à la graphie <un>. Dans les cas de Paris et du Québec, cela représente 9 contextes dans la liste de mots PFC, 6 contextes dans la liste de mots complémentaire et 6 contextes dans le texte PFC. Dans le cas de la Suisse romande, les nombres septante-et-un et nonante-et-un s'ajoutent aux 9 autres contextes dans la liste de mots PFC, pour un total de 11 contextes dans cette liste, les 6 contextes de la liste de mots complémentaire et les 6 contextes du texte PFC étant les mêmes que pour Paris et le Québec. Ainsi, avec 20 locuteurs par région, 420 contextes auraient théoriquement dus être codés dans les cas de Paris et du Québec (300 dans les listes de mots et 120 dans le texte PFC) et 460 dans le cas de la Suisse romande (340 dans les listes de mots et 120 dans le texte PFC), pour un total de 1300 contextes. Cependant, une occurrence étant manquante dans la lecture de la liste de mots PFC d'un des locuteurs suisses romands, ${ }^{10}$ le nombre effectif de codages s'élève à 1299 .

Alors que pour Paris et la Suisse romande, cette opposition est la seule à avoir été testée au niveau des voyelles, le cas du Québec est plus complexe, car la réalisation phonétique du /ã/ et celle du / $\tilde{\varepsilon} /$ ont également été prises en compte. Concernant la réalisation de la voyelle nasale /ã/, le codage s'est appuyé sur celui proposé dans l'étude de Remysen (2016a), qui a utilisé un codage tenant compte $\mathrm{du}$ « [. . . ] caractère graduel des différentes réalisations de la nasale » (Remysen 2016a, 143). Le codage quinaire de Remysen (2016a), qui comprenait les variantes

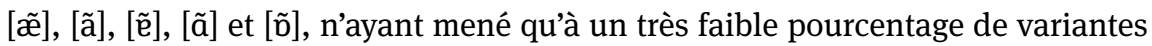

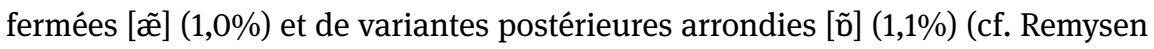
2016a, 144), je l'ai réduit pour le présent ouvrage à un codage ternaire, qui com-

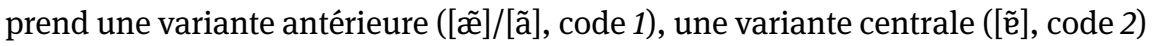

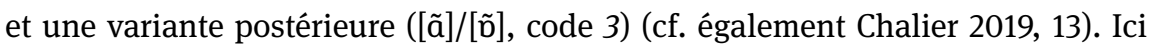
aussi, le codage a été effectué dans tous les contextes correspondant à un /ã/, c'est-à-dire tous les contextes correspondant aux graphies <an> et <en> lorsque celles-ci sont prononcées /ã/ selon la prononciation de référence. Cela représente 55 contextes dans la liste de mots PFC, 26 contextes dans la liste de mots complémentaire et 50 dans le texte PFC, pour un total théorique de 131 codages par locuteur et, compte tenu des 20 locuteurs-modèles québécois, 2620 codages dans

10 Occurrence manquante : locuteur slRas1 : le locuteur commence la lecture de la liste de mots PFC sans lire le chiffre $\underline{1}$ (/õ̃/ / $\tilde{\varepsilon} /$ ). 
le corpus global. Cependant, trois occurrences étant manquantes ${ }^{11}$ et une occurrence répétée, ${ }^{12}$ leur nombre effectif s'élève à 2618 (1620 dans les mots isolés des deux listes de mots, 998 dans le texte PFC).

Dans le cas du / $\tilde{\varepsilon} /$, le codage a été réduit à un codage binaire comprenant

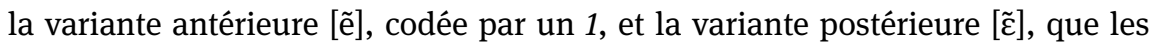
codeurs signalaient par un 2. Dans ce cas également, les codages ont été effectués dans tous les contextes correspondant à un / $\tilde{\varepsilon} /$, c'est-à-dire les contextes correspondant aux graphies $<$ in $>,<\mathrm{im}\rangle,\langle\mathrm{ym}\rangle$ et $<$ en $>$ lorsque celles-ci sont prononcées / $\tilde{\varepsilon} /$ selon la prononciation de référence. Cela représente 51 contextes dans la liste de mots PFC, 17 contextes dans la liste complémentaire et 19 contextes dans le texte PFC. Comme il y a 20 locuteurs-modèles québécois, il s’agit ainsi théoriquement de 87 contextes par locuteur et de 1740 contextes au total. Cependant, une occurrence étant manquante, ${ }^{13}$ deux étant répétées ${ }^{14}$ et une ajoutée, ${ }^{15}$ le nombre effectif de contextes s'élève 1742 (1361 dans les mots isolés des deux listes de mots, 381 dans le texte PFC) (cf. tableau 24).

Tableau 24 : Système de codage des voyelles nasales analysées dans le corpus.

\begin{tabular}{|c|c|c|c|}
\hline Oppositions & Codages & Exemple & \\
\hline \multirow[t]{2}{*}{ 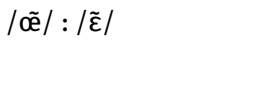 } & 1 = voyelle arrondie [œ̃] & brun & [вьюе̃] \\
\hline & $2=$ voyelle non arrondie $[\tilde{\varepsilon}]$ & brin & {$[\mathrm{b}\llcorner\tilde{\varepsilon}]$} \\
\hline \multirow[t]{3}{*}[\tilde{\dddot{x}}]{$/[\tilde{a}] \sim[\tilde{\mathrm{e}}] \sim[\tilde{a}] /[\tilde{\mathrm{p}}]$} & $1=$ voyelle antérieure [æ̃]/[ã] & présent & [рвеzã] \\
\hline & $2=$ voyelle centrale $[\tilde{e}]$ & & [рвеzе̃] \\
\hline & $3=$ voyelle postérieure [ã] $/[\tilde{\mathrm{p}}]$ & & [рвеzã] \\
\hline \multirow[t]{2}{*}[\tilde{e}]{$\sim[\tilde{\varepsilon}]$} & 1 = voyelle antérieure [ẽ] & cinq & [sẽk] \\
\hline & $2=$ voyelle postérieure $[\tilde{\varepsilon}]$ & & {$[\mathrm{s} \tilde{\varepsilon} \mathrm{k}]$} \\
\hline
\end{tabular}

11 Occurrences manquantes : locuteur cmRbl1 : /ã/ dans trente-six de la liste de mots PFC (le locuteur saute ce chiffre dans sa lecture); locuteur cmTad1 : /ã / dans il en dira dans le texte PFC (le locuteur saute ce mot dans sa lecture) ; locuteur cmTvm1 : /ã/ dans dans la tournée dans le texte PFC (le locuteur saute ce mot dans sa lecture).

12 Occurrence répétée : cqRgl1 : /ã/ dans penser dans la liste de mots complémentaire.

13 Occurrence manquante : locuteur cmTmm 1 : / $\tilde{\varepsilon} /$ dans cinquante-deux de la liste de mots PFC (le locuteur remplace cinquante-deux par quarante-deux dans sa lecture).

14 Occurrences répétées : locuteur cqRgl1 : / $\tilde{\varepsilon} /$ dans quatre-vingt-neuf et quatre-vingt-dix (le locuteur répète les deux entrées dans sa lecture).

15 Occurrence ajoutée : locuteur cmRbl1 : / $\tilde{\varepsilon} /$ dans fin de citation (le locuteur ajoute cette expression après la citation placée entre guillemets dans le texte PFC). 
Après cette phase de codage, les données ont été extraites à l'aide de l'outil informatique DOLMEN (cf. Eychenne/Paternostro 2016) et analysées statistiquement

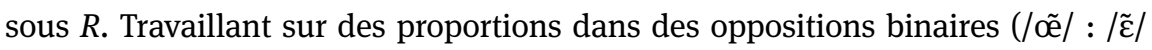

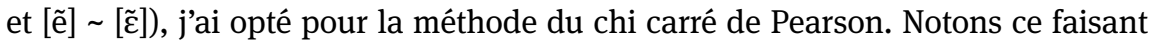
que si l'opposition $[\tilde{e}] /[\tilde{a}] \sim[\tilde{e}] \sim[\tilde{a}] /[\tilde{b}]$ codée dans le cas du Québec est théoriquement de nature catégorielle ordinale (codage 1 pour antérieur, codage 2 pour central et codage 3 pour postérieur), la variante antérieure [ã]/[ã] n’atteint, dans mes données (cf. 5.3.2.2), jamais un nombre d'observations suffisant pour être prise en compte dans les modèles statistiques. C'est la raison pour laquelle le chi

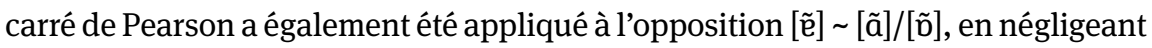
dans les tests statistiques les quelques observations de la variante [æ̃]/[ã].

\subsection{Perception : évaluation des voyelles des « locuteurs- modèles " par des auditeurs « non experts "}

La troisième méthode utilisée dans le cadre du présent ouvrage concerne les perceptions. Il s'agit de tests de perception effectués dans chacune des trois régions afin de mettre en lumière les attitudes perceptives d'auditeurs non experts face aux voyelles caractéristiques de l'usage des locuteurs-modèles de ces régions. L'objectif de ces tests de perception était ainsi de découvrir dans quelle mesure les voyelles spécifiques à chacune des régions sont privilégiées ou non par les auditeurs de ces régions par rapport à la prononciation de référence équivalente (p. ex. année : [ane:] en tant que prononciation suisse romande vs. [ane] en tant que prononciation de référence).

Le test se base sur un total de 53 paires de stimuli (prononciation régionale vs. prononciation de référence), évalués par 96 informateurs par région (288 au total). Les questions posées par rapport à ces stimuli touchent à trois catégories : premièrement, le degré de ce que la dialectologie populaire nomme correctness (cf. Preston 1999b, xxxiv), deuxièmement, la prononciation idéale des présentateurs de journaux de ces régions et, troisièmement, la prononciation qui, selon ces informateurs, devrait être enseignée dans les cours de FLE . Le test a été effectué sur la plateforme SoSci Survey (cf. SoSci Survey GmbH 2018), spécialement conçue pour les enquêtes scientifiques.

La présentation détaillée de cette méthode sera structurée en quatre parties : tout d'abord, j'expliquerai le choix des stimuli sur lesquels repose le test de perception (cf. 3.5.1). Ensuite, je présenterai les trois types de questions posées et les catégories de réponses ainsi que leur pertinence pour les objectifs du test (cf. 3.5.2). En un troisième temps, j’aborderai la sélection et le recrutement des informateurs (cf. 3.5.3). Pour finir, je présenterai le protocole d'analyse statistique des données (cf. 3.5.4). 


\subsubsection{Stimuli}

Les tests de perception sont basés sur la sélection de syntagmes et de mots lus déjà pris en compte dans les analyses de production (cf. tableaux 18-23, chapitre 3.4.2.1). Cependant, dans le cas des tests de perception, de manière similaire à la procédure de Detey/Le Gac (2008, 480), seuls les stimuli des mots contenant les traits considérés comme "régionaux » ont été retenus pour chacune des paires, avec pour chaque mot un stimulus donné par un locuteur lisant le mot avec ce trait régional ainsi qu'un stimulus d'un autre locuteur lisant le mot avec le trait de référence correspondant. Prenons, en guise d'exemple, l'opposition /a/ : /a/ dans des mots isolés lus par des présentateurs suisses romands. Alors que les analyses de production portent sur tous les stimuli enregistrés pour les deux mots de la paire minimale patte $~$ pâte, le test de perception ne prend en compte que deux des stimuli des lectures de pâte, l'un d'entre eux étant lu [pat] par un locuteur et l'autre [pat] par un autre locuteur. Cet exemple est résumé dans le tableau 25 :

Tableau 25 : Stimuli pris en compte : différences entre les analyses de production et les tests de perception à l'exemple de la paire minimale patte $\sim$ pâte.

\begin{tabular}{|c|c|c|c|}
\hline Paire minimale & $\begin{array}{l}\text { Productions } \\
\text { des locuteurs }\end{array}$ & $\begin{array}{l}\text { Analyses de } \\
\text { production }\end{array}$ & Tests de perception \\
\hline patte $\sim$ pâte & $\begin{array}{l}\text { patte : [pat] } \\
\text { pâte : } \\
\text { [pat] } \sim \text { [pat] }\end{array}$ & $\begin{array}{l}\text { Analyse de tous } \\
\text { les stimuli existant } \\
\text { pour patte et pâte }\end{array}$ & $\begin{array}{l}\text { Prise en compte de deux des stimuli } \\
\text { du mot contenant le trait « régional »: } \\
\text { pâte }[\text { pat] } \sim \text { [pat] }\end{array}$ \\
\hline
\end{tabular}

Ce choix repose sur le fait que les auditeurs ont été invités à évaluer les prononciations entendues sur la base d'une représentation graphique du mot qu'ils voyaient à l'écran, cette représentation graphique étant justement le mot contenant le trait considéré comme « régional » (p. ex. pâte dans l'exemple ci-dessus). Ainsi, étant donnée cette représentation graphique, je n’ai eu besoin de prendre en compte que les lectures de cet unique mot.

De même que pour les analyses de production, notons qu'il était nécessaire ici aussi que les stimuli soient lus : il me fallait en effet contrôler au mieux que ce sont bien les traits de prononciation devant être testés qui sont évalués par les auditeurs (cf. Woehrling/Boula de Mareüil 2005, 91-92). Ainsi, j'ai choisi, d'une part, de présenter les paires de voyelles dans un mot isolé prononcé une fois avec la voyelle de référence (p. ex. brin [bьर̃ ] dans le test québécois) et une fois avec la voyelle considérée comme régionale (p. ex. brin [bsẽ] dans ce même test). Cette méthode garantissait en effet au mieux que les auditeurs se réfèrent bien à cette 
différence de timbre dans leur évaluation. Le test a, d'autre part, également pris en compte des syntagmes plus longs tirés du texte lu (p. ex. l'extrait Le village de Beaulieu est en grand émoi pour tester l'opposition $/ \varepsilon /: / \mathrm{e} /$ ), en raison de leur nature moins artificielle que des mots isolés, combinée à leur meilleure comparabilité par rapport à des stimuli de nature spontanée (cf. Woehrling/Boula de Mareüil 2005, 91-92).

Soulignons cependant que la nature des stimuli pour des tests de perception reste une question controversée : alors que les stimuli lus sont mieux contrôlables et comparables, mais également artificiels, les stimuli non lus sont plus proches de l'usage spontané des locuteurs (cf. Purschke 2011, 151), mais permettent une comparaison moins fiable des données. Notons pourtant que Postlep $(2010,106)$ obtient des résultats contredisant ces observations : ses résultats liés à des stimuli lus présentent en effet un écart-type plus élevé que ceux touchant à des stimuli de nature spontanée.

En résumé, chaque trait mentionné dans les tableaux 18-23 (cf. 3.4.2.1) a été inséré dans le test de perception selon le schéma du tableau 26, et ce, dans la mesure du possible, aussi bien sous forme de paires de mots que sous forme de paires de syntagmes. Certains traits n'apparaissent néanmoins pas dans les listes de mots ou dans le texte et n'ont donc pu être pris en compte que dans l'un ou l'autre contexte.

Tableau 26 : Choix des stimuli.

\begin{tabular}{llll}
\hline Sources & Types de stimuli & Paires de mots testées \\
\hline Extraits du texte PFC & Phrases ou & par & prononciation régionale vs. \\
& syntagmes & paires & prononciation de référence \\
\hline Extraits des listes de mots & Mots isolés & & \\
\hline
\end{tabular}

Le choix concret de paires de stimuli à utiliser dans les tests de perception a été effectué selon les critères suivants :

- Dans le cas des différences de timbre de voyelles orales (p. ex. pâtes [pat] $\sim$ [pat]), le choix s'est fait sur la base de la distance acoustique entre les voyelles, chaque paire de mots prise en compte contenant ainsi les deux réalisations de la voyelle en question les plus éloignées l'une de l'autre selon les formants $F_{1}$ et $F_{2}$. Ce choix repose sur l'hypothèse de Green/Gail/Kuhl (1997, 686-687), selon laquelle une distance acoustique plus élevée dans la production est corrélée avec une meilleure distinction dans la perception. Dans le cas spécifique des diérèses et des synérèses, le choix des stimuli à prendre en compte s'est fait sur la base d'une lecture qualitative de spectrogramme (cf. 3.4.2.2.2), avec comme principal critère une structure formantique instable 
et en variation constante pour les synérèses et une structure à deux voyelles présentant une partie stable pour les diérèses.

- Dans le cas des différences de durée (p. ex. année [ane] [ane:]), le choix s'est fait sur la base des mesures de longueur des voyelles, chaque paire de mots prise en compte contenant les voyelles les plus brèves et les plus longues mesurées dans le sous-corpus respectif (c'est-à-dire soit le sous-corpus suisse romand, soit le sous-corpus québécois, étant donné qu'aucune opposition de durées n’a été testée dans le sous-corpus parisien).

- Dans le cas des différences de timbre de voyelles nasales (p. ex. brun /bьо̃ / /bь $\tilde{\varepsilon} /$ ), le choix s'est fait sur la base d'une appréciation auditive des codeurs de chaque sous-corpus, le choix s'étant, ici aussi, porté sur des stimuli particulièrement bien distinguables l'un de l'autre. En effet, tout comme dans le cas de l'analyse de production des voyelles nasales (cf. 3.4.2.3), un choix basé sur une analyse acoustique n'aurait été que peu fiable en raison des différents effets acoustiques de la nasalisation (transitions des formants, augmentation de la largeur des bandes de formants, antiformants).

Notons finalement qu'afin d'éviter toute influence due à la différence entre les voix masculines ou féminines sur les évaluations, toutes les paires de stimuli utilisées dans le cadre des trois tests sont attribuables à des hommes. Ce choix repose sur le fait que les hommes sont surreprésentés dans certaines chaînes (cf. 3.4.1.1), ce qui a permis de choisir plus facilement des stimuli adéquats, certaines paires de mots ou de syntagmes ne pouvant pas être trouvées pour les deux sexes. Certaines voyelles ont en effet été réalisées par toutes les locutrices avec un timbre très similaire pouvant être attribué à la même voyelle au niveau acoustique, l'autre voyelle étant donc manquante pour la globalité de ce groupe de locutrices. Mentionnons en guise d'exemple le mot pâte, réalisé systématiquement par toutes les locutrices parisiennes comme [pat], ce qui aurait rendu la comparaison des prononciations [pat] et [pat] impossible si seules des voix féminines avaient été prises en compte.

\section{Paris}

Dans le cas de Paris, étant donnée la proximité considérable entre la prononciation de référence (prononciation décrite dans le chapitre 2.3.1) et la prononciation des locuteurs-modèles parisiens, j'ai également pris en compte des stimuli de locuteurs " ordinaires » nés à Paris (tirés du corpus de Pustka 2007) et j'en ai comparé les résultats avec ceux obtenus avec des stimuli de locuteurs-modèles. L'objectif de cet ajout était de découvrir si une différence est faite entre ces deux groupes de locuteurs dans la perception des auditeurs. 
Les stimuli pris en compte dans le cadre du test de perception effectué à Paris sont listés dans le tableau 27.

Tableau 27 : Stimuli pris en compte dans le test de perception effectué à Paris.

\begin{tabular}{|c|c|c|c|c|c|}
\hline & \multirow{2}{*}{$\begin{array}{l}\text { Traits parisiens } \\
\text { évalués }\end{array}$} & \multicolumn{2}{|c|}{ Mots isolés } & \multicolumn{2}{|c|}{ Syntagmes } \\
\hline & & $\begin{array}{l}\text { Locuteurs- } \\
\text { modèles }\end{array}$ & $\begin{array}{l}\text { Locuteurs } \\
\text { ordinaires }\end{array}$ & $\begin{array}{l}\text { Locuteurs- } \\
\text { modèles }\end{array}$ & $\begin{array}{l}\text { Locuteurs } \\
\text { ordinaires }\end{array}$ \\
\hline 1. & $\begin{array}{l}\text { Maintien de } \\
\text { l'opposition } \\
\text { /a/ : /a/ }\end{array}$ & \multicolumn{2}{|c|}{ pâte : [pat] [pat] } & \multicolumn{2}{|c|}{$\begin{array}{l}\text { Et plus récemment, son usine de } \\
\text { pâtes italiennes : [pat] } \sim \text { [pat] }\end{array}$} \\
\hline 2. & $\begin{array}{l}\text { Neutralisation } \\
\text { de l'opposition } \\
/ \mathrm{e} /: \mid \varepsilon /\end{array}$ & \multicolumn{2}{|c|}{ épais : [epe] [epع] } & \multicolumn{2}{|c|}{ 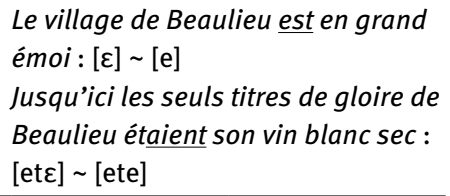 } \\
\hline 3. & $\begin{array}{l}\text { Neutralisation } \\
\text { de l'opposition } \\
/ \varnothing /: / œ /\end{array}$ & \multicolumn{2}{|c|}{ jeûne : [zœn] [3øn] } & \multicolumn{2}{|c|}{$\begin{array}{l}\text { Quelques fanatiques auraient même } \\
\text { entamé un jeûne prolongé dans } \\
\text { l'église de Saint Martinville: } \\
\text { [उœn] [उøn] }\end{array}$} \\
\hline 4. & $\begin{array}{l}\text { Neutralisation } \\
\text { de l'opposition } \\
/ \tilde{\varepsilon} /: / \tilde{e} /\end{array}$ & \multicolumn{2}{|c|}{ 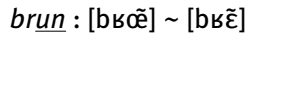 } & \multicolumn{2}{|c|}{ D’un autre côté : [ø̃] $~[\tilde{\varepsilon}]$} \\
\hline
\end{tabular}

Par rapport à $/ \mathrm{e} /: / \varepsilon /$, rappelons que l'opposition aura tendance à se maintenir dans les paires minimales été étai(en)t et épée épais, alors que dans la forme est, la tendance sera à la fermeture en [e] (cf. 3.4.2.1), ces évolutions pouvant également avoir des répercussions sur la perception des auditeurs.

Par ailleurs, en ce qui concerne l'opposition / $\tilde{\varepsilon} /:$ / ẽ/, étant donné qu'en production le [œẽ] aura tendance à mieux se maintenir dans brun que dans un (cf. 3.4.2.1), il est probable qu'une éventuelle tendance vers le [ $\tilde{\varepsilon}]$ soit évaluée différemment dans ces deux mots lors du test de perception.

$\mathrm{Au}$ total, 18 paires de stimuli - soit 36 stimuli individuels répartis sur les deux types de locuteurs (locuteurs-modèles et locuteurs " ordinaires ») - ont donc été prises en compte dans le cadre de ce test de perception, 8 de ces paires de stimuli étant des mots isolés et 10 des syntagmes.

\section{Suisse romande}

Dans le cas de la Suisse romande, le problème de la grande proximité entre le modèle traditionnel de référence et les variétés suisses romandes se pose moins 
et seuls des locuteurs-modèles ont donc été pris en compte. Notons que dans le cas de l'opposition /e/ $: / \varepsilon /$ dans sa fonction de distinction de certains temps et modes en syllabe finale ouverte, aucune paire de syntagmes n'a pu être trouvée dans le texte PFC. Le trait n'a donc été testé que sur la base de mots isolés. Mentionnons par ailleurs qu'aucune diphtongaison n'a pu être relevée dans les analyses de production des mots année [ane:'] (texte lu) et pensée [pãse: (liste de mots complémentaire), et que de ce fait, aucun stimulus présentant cette caractéristique n’a pu être inséré dans le test de perception (cf. tableau 28).

Tableau 28 : Stimuli pris en compte dans le test de perception effectué en Suisse.

\begin{tabular}{|c|c|c|c|}
\hline & Traits suisses romands évalués & Mots isolés & Syntagmes \\
\hline 1. & $\begin{array}{l}\text { Opposition quantitative } \\
\text { en syllabe finale fermée + } \\
\text { différence de timbre }\end{array}$ & $\begin{array}{l}\text { pâate : } \\
\text { [pat] } \sim \text { [pat] }\end{array}$ & $\begin{array}{l}\text { Et plus récemment, son usine de } \\
\text { pâtes italiennes : [pat] [pat] }\end{array}$ \\
\hline 2. & $\begin{array}{l}\text { Opposition quantitative en } \\
\text { syllabe finale ouverte }\end{array}$ & $\begin{array}{l}\text { pensée } \\
\text { [pãse] [pãse:] }\end{array}$ & $\begin{array}{l}\text { Syntagme : En fin d'année : } \\
\text { [ane] [ane:] }\end{array}$ \\
\hline 3. & $\begin{array}{l}\text { Opposition } / e /: / \varepsilon / \text { en syllabe } \\
\text { finale ouverte } \\
\text { (distinction de certains temps et } \\
\text { modes) }\end{array}$ & $\begin{array}{l}\text { je pourrai } \\
\text { [риве] } \sim \text { [ривє] }\end{array}$ & (aucun stimulus) \\
\hline 4. & 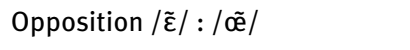 & 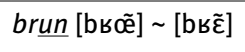 & 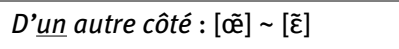 \\
\hline 5. & $\begin{array}{l}\text { Préférence de la diérèse à la } \\
\text { synérèse }\end{array}$ & $\begin{array}{l}\text { muette } \\
{[\mathrm{my} . \varepsilon \mathrm{t}] \sim[\mathrm{mu} \varepsilon \mathrm{t}]}\end{array}$ & 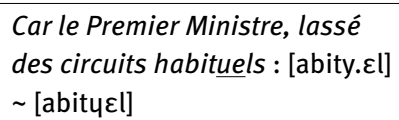 \\
\hline
\end{tabular}

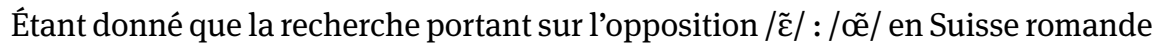
observe que le /õ/ semble être, contrairement au cas de Paris, plus résistant dans un que dans brun (cf. 3.4.2.1), on peut ici aussi s'attendre à ce que l'évaluation de

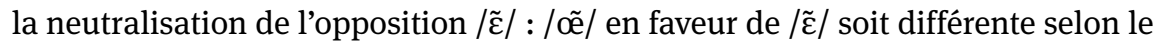
mot, mais dans le sens inverse de l'évolution en cours à Paris. Notons aussi que la question de l'influence de la variation lexicale devra également être abordée dans la présentation des évaluations touchant à l'opposition quantitative dans pensée année et à la diérèse/synérèse dans muette habituels.

Au total, 9 paires de stimuli, soit 18 stimuli individuels, ont été prises en compte dans le cadre du test de perception effectué en Suisse romande, 5 de ces paires de stimuli étant des mots isolés et 4 des syntagmes. 


\section{Québec}

Le cas du Québec est similaire à celui de la Suisse, un seul groupe de locuteurs ayant été pris en compte, celui des locuteurs-modèles. Précisons que pour réduire le plus possible la durée du test, deux traits présentés dans le chapitre 2.3.4 ont été réunis en une double paire de voyelles. Il s'agit de l'opposition quantitative $/ \varepsilon /: / \varepsilon: /(c f$. tableau 23, trait 3) et de la diphtongaison des voyelles allongées (cf. tableau 23, trait 8), rassemblées dans la double paire de voyelles à l'intérieur du mot fête ([fct] [fєtt] [fa $\left.{ }^{3} \mathrm{t}\right]$ ) (cf. tableau 29, trait 3). Mentionnons par ailleurs qu'aucune équivalence à ces deux traits n'a pu être trouvée dans le texte PFC, raison pour laquelle ils n'ont pu être testés que sur la base de mots isolés. De manière similaire, dans le cas du dévoisement (ou effacement) des voyelles /i, y, u/, aucune paire de stimuli convenables n'a pu être trouvée dans les mots isolés. Soulignons finalement que dans le cas de la réalisation de /ã/, seules deux variantes ont pu être prises en compte - la variante centrale [ẽ ] et la variante postérieure [ã] - étant donné qu'aucune variante antérieure [ã] utilisable n’a pu être trouvée dans les contextes testés dans les productions des locuteurs-modèles (cf. tableau 29).

Tableau 29 : Stimuli pris en compte dans le test de perception effectué au Québec.

\begin{tabular}{|c|c|c|c|}
\hline & Traits québécois évalués & Mots isolés & Syntagmes \\
\hline 1. & Opposition /a/: /a/ & $\begin{array}{l}\text { pâte : } \\
\text { [pat] [pat] }\end{array}$ & $\begin{array}{l}\text { Et plus récemment, son usine } \\
\text { de pâates italiennes: [pat] } \\
\text { [pat] }\end{array}$ \\
\hline 2. & /a/ final dans <-oi> : [wa] ou [wa] & 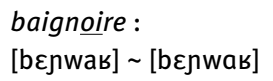 & $\begin{array}{l}\text { trois échevins : } \\
\text { [tвwa] [tвwa] }\end{array}$ \\
\hline 3. & $\begin{array}{l}\text { Opposition quantitative } / \varepsilon / \\
\text { vs. } / \varepsilon: /+ \text { diphtongaisons des } \\
\text { voyelles allongées }\end{array}$ & $\begin{array}{l}\text { fête: } \\
{[f \varepsilon t] \sim[f \varepsilon: t] \sim\left[\mathrm{fa}^{3} \mathrm{t}\right]}\end{array}$ & (aucun stimulus) \\
\hline 4. & Quatre voyelles nasales & 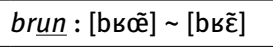 & 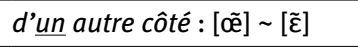 \\
\hline 5.1 & Réalisation de / $\tilde{\varepsilon} /$ en [ẽ] & $\begin{array}{l}\text { médecin : } \\
{[\text { meds } \tilde{\varepsilon}] \sim[\text { medsẽ] }}\end{array}$ & 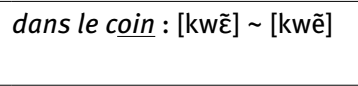 \\
\hline 5.2 & $\begin{array}{l}\text { Réalisation de /ã/ en [ẽ] (central) } \\
\text { ou [ã] postérieur }\end{array}$ & $\begin{array}{l}\text { penser: } \\
\text { [pẽse] } \sim \text { [pãse] }\end{array}$ & $\begin{array}{l}\text { l'ancienne poste vendue: } \\
\text { [vẽdy] [vãdy] }\end{array}$ \\
\hline 6. & $\begin{array}{l}\text { Ouverture et relâchement des } \\
\text { voyelles /i, y, u/ }\end{array}$ & $\operatorname{six}:[\mathrm{sis}] \sim[\mathrm{sıs}]$ & $\begin{array}{l}\text { autour des mêmes villes: } \\
\text { [vil] [vil] }\end{array}$ \\
\hline 7. & $\begin{array}{l}\text { Dévoisement (ou effacement) } \\
\text { des voyelles /i, y, u/ }\end{array}$ & (aucun stimulus) & $\begin{array}{l}\text { des vérifícations d'identité : } \\
\text { [vesifikasj̄̃] [vesif:kasjõ] }\end{array}$ \\
\hline
\end{tabular}

Concernant l'opposition /œẽ/: / / $/$ /, le problème de variation des mots utilisés (brun dans le cas des mots isolés, un dans le cas des syntagmes) se pose également pour 
le Québec. Cependant, étant donnée la stabilité de l'opposition reconnue unanimement dans la littérature (cf. 2.3.4), la différence d'évaluation des réalisations du /õ̃/ dans les deux mots devrait s'avérer moins élevée que dans les cas de Paris et de la Suisse romande. Il faudra également, tout comme dans les analyses de production, porter attention à la variation lexicale pour $/ \tilde{\varepsilon} /$ dans médecin $\sim$ coin, /ã/ dans penser $\sim$ vendue et /i/ dans six $\sim$ ville.

Ainsi, 13 paires de stimuli - qui correspondent à 26 stimuli individuels - et une double paire de stimuli $\left(\underline{\hat{e}} t e[\mathrm{f} \varepsilon \mathrm{t}] \sim[\mathrm{f} \varepsilon \mathrm{t}] \sim\left[\mathrm{fa}^{3} \mathrm{t}\right]-\right.$ correspondant à 3 stimuli individuels - ont été prises en compte dans le cadre du test de perception effectué au Québec, pour un total de 29 stimuli individuels. Six de ces paires, ainsi que la double paire, sont des mots isolés, les 7 autres paires des syntagmes.

\subsubsection{Questions et catégories de réponses}

La tâche d'évaluation des différentes prononciations des voyelles présentes dans ces paires de mots ou de syntagmes a été élaborée de la façon suivante : les auditeurs, qui voyaient la représentation graphique du mot à l'écran en même temps qu'ils en entendaient l'une des deux prononciations, étaient invités à évaluer la prononciation entendue. Notons ici que la présentation graphique peut être problématique, étant donné qu'elle peut pousser les informateurs à chercher une différence de prononciation qu'ils n'entendraient peut-être pas dans un contexte sans influence de la graphie. Cette variante avec présentation graphique du mot a été cependant choisie afin d'expliciter au mieux l'item à évaluer, la simple évaluation sans support graphique pouvant, dans la perspective inverse, mener à des confusions quant à l'objet exact de l'évaluation.

L'évaluation a été effectuée sur la base de trois types de questions, qui avaient pour objectif de tester trois types de normes (cf. 2.1.1). Ces trois types de normes reposent sur deux conceptions théoriques fondamentalement différentes des attitudes : il existe, premièrement, une conception des attitudes issue de la tradition de la technique du locuteur masqué (matched-guise, cf. Lambert et al. 1960), qui les considère comme des entités mentales stables et indépendantes $d u$ contexte dans lequel elles apparaissent (cf. Hartley 1996 ; Preston 1996 ; Hartley 1999 ; Kuiper 1999 ; Preston 1999a). Selon une autre conception en revanche, elles sont dépendantes des situations de communication dans lesquelles elles sont exprimées (cf. Hyrkstedt/Kalaja 1998 ; Tophinke/Ziegler 2006 ; Soukup 2014). Ces deux approches peuvent être reportées au domaine des normes. En effet, Pustka/ Chalier/Jansen (2017) font une différence entre les normes descriptives des productions, qui sont dépendantes des situations de communication, et la ou les norme(s) prescriptive(s) des représentations, bien plus homogènes, les locuteurs 
ayant une conscience relativement nette de ce qui peut être considéré comme un " bon » ou un " mauvais » usage linguistique (cf. Pustka/Chalier/Jansen 2017, 102-103). Ainsi, les trois types de questions formulées dans le cadre du test de perception testent ces deux types de norme.

\section{Question 1 : évaluation (sans contexte explicite)}

La première question (cf. figure 10) est posée délibérément sans mention explicite de quelque contexte que ce soit, afin de tester la conception d'une norme stable et indépendante de la situation de communication.

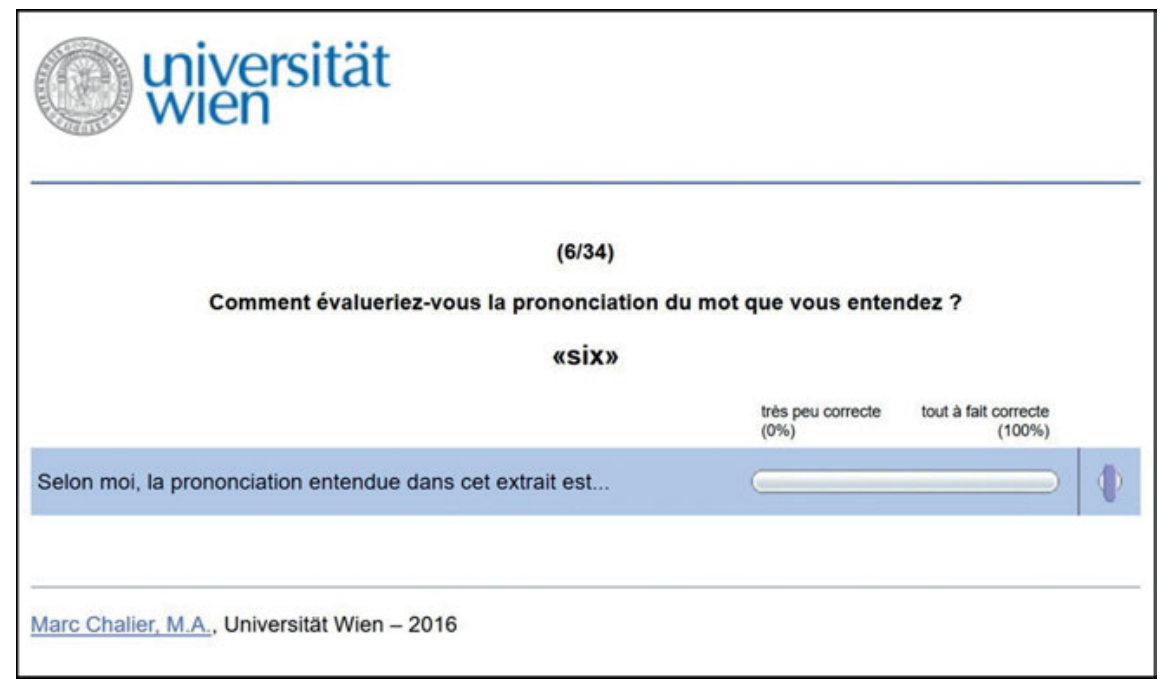

Figure 10 : Question 1 : Comment évalueriez-vous la prononciation du mot/groupe de mots que vous entendez ?; exemple du stimulus six [sıs] (Québec) ${ }^{16}$.

Les deux questions suivantes sont, pour leur part, posées en mentionnant des contextes précis pour cette norme afin de tester la conception d'une norme plurielle dépendante de la situation de communication. Par ailleurs, ces deux questions touchent à deux domaines dans lesquels la question d'une norme de référence est particulièrement pertinente (cf. déjà Chalier 2018).

16 La question des catégories de réponse est abordée plus bas (cf. infra). 


\section{Question 2 : prononciation idéale des présentateurs de journaux}

La première de ces questions (cf. figure 11) aborde la thématique de la prononciation idéale d'un présentateur de télévision à Paris, en Suisse romande et au Québec.

(2/16)

Selon vous, comment un présentateur de télévision québécois devrait-il prononcer le mot suivant ?

«six»

Comme dans le 1er enregistrement

Comme dans le 2ème enregistrement

Je ne sais pas

Marc Chalier, M.A., Universităt Wien - 2016

Figure 11 : Question 2 : Selon vous, comment un présentateur de télévision suisse/québécois/ parisien devrait-il prononcer le mot/le groupe de mots suivant? ; exemple des stimuli six [sis] vs. six [sıs] (Québec).

\section{Question 3 : prononciation à enseigner dans les cours de FLE}

La deuxième de ces questions à contexte (cf. figure 12) touche, pour sa part, au modèle de prononciation à enseigner dans les cours de FLE en immersion dans ces trois régions.

\section{Catégories de réponses}

Ces trois questions ont été combinées à différentes catégories de réponses. Dans le cas de la première question (Question 1), les auditeurs ont été invités à évaluer les stimuli en plaçant un curseur sur une échelle allant de $0 \%$ à $100 \%$ quant au degré de correctness des stimuli ( $0 \%$ : prononciation très peu correcte ; $100 \%$ : prononciation tout à fait correcte) (cf. figure 10). Ce faisant, contrairement aux cas des questions 2 et 3, chaque stimulus a été évalué individuellement. Cette échelle présente l'avantage pour les auditeurs de pouvoir transformer leurs impressions en une quantité visuelle de façon spontanée sans avoir à choisir entre des catégo- 


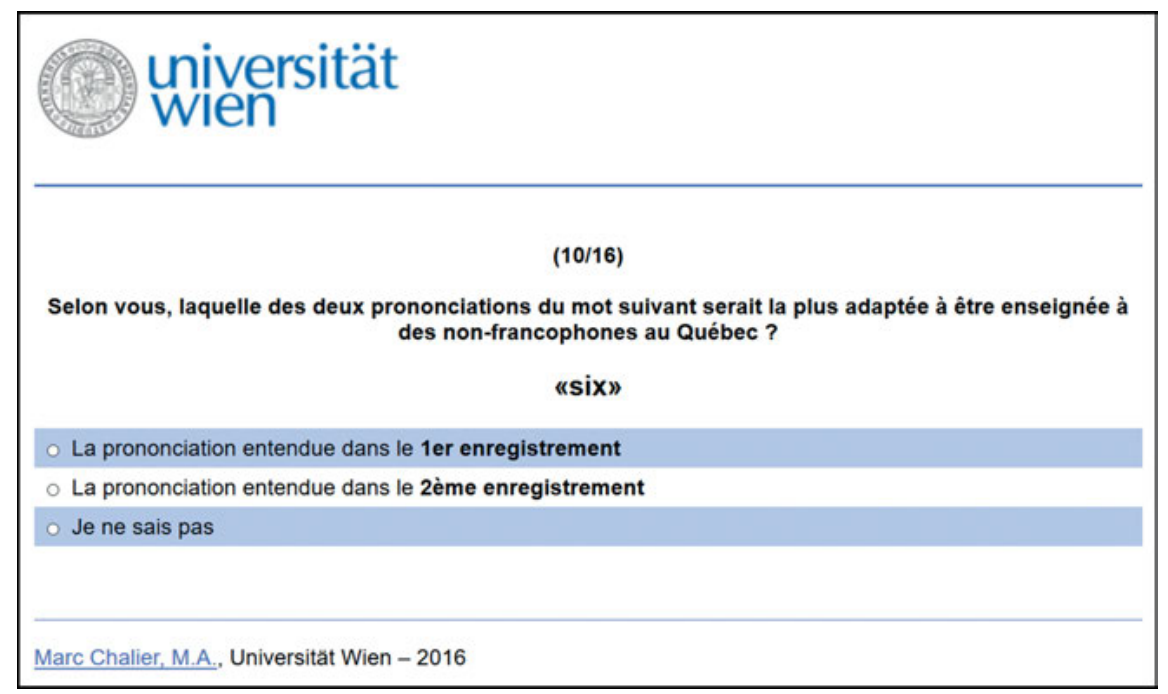

Figure 12 : Question 3 : Selon vous, laquelle des deux prononciations du mot/de la phrase suivant(e) serait la plus adaptée à être enseignée à des non-francophones à Paris/en Suisse romande/au Québec ?; exemple des stimuli six [sis] vs. six [sıs] (Québec).

ries trop évidentes (cf. Porst $\left.{ }^{2} 2009,92\right)$. Pour les questions 2 et 3, j’ai opté pour des réponses à choix simple parmi deux possibilités de réponse (ou trois dans le cas de la double paire de voyelles dans le mot fête $:[\mathrm{fct}] \sim$ [fєtt] [fa $\left.{ }^{3} \mathrm{t}\right]$ ) (cf. figures 11 et 12). Les informateurs étaient donc invités, après avoir écouté les deux (ou trois) stimuli, à cliquer sur le stimulus qu'ils privilégient pour les présentateurs de télévision et pour le domaine de l'enseignement du FLE dans leur région. Notons qu'il ne s'agissait pas de questions à choix forcé, étant donné que les informateurs avaient également la possibilité de choisir une catégorie « je ne sais pas ». Le tableau 30 présente un résumé de la combinaison des trois types de questions avec les deux types de réponses utilisés.

À propos de la structure du questionnaire, notons que la sélection des informateurs s'est faite sur la base d'un échantillonnage par quotas limité à 96 informateurs par région et que leur nombre dans chaque groupe était donc trop faible pour pouvoir procéder à une randomisation. Ainsi, tous les informateurs ont répondu à la totalité des questions, les questions et leurs catégories de réponse apparaissant systématiquement dans le même ordre. Les résultats statistiques des tests de perception sont donc à considérer avec précaution : du fait de ce renoncement à toute randomisation, d'éventuels facteurs de confusion biaisant les résultats ne peuvent être exclus, ce qui peut avoir un effet sur la validité des résultats (cf. Drager 2018, 44). 
Tableau 30 : Questions et catégories de réponse du test de perception.

\begin{tabular}{lll}
\hline Type de question & Question & Réponses \\
\hline $\begin{array}{l}\text { Évaluations } \\
\text { (sans contexte } \\
\text { explicite) }\end{array}$ & $\begin{array}{l}\text { Comment évalueriez-vous la prononciation du } \\
\text { groupe de mots que vous entendez ? }\end{array}$ & Échelle 0\%-100\% \\
\hline $\begin{array}{l}\text { Présentateurs de } \\
\text { télévision }\end{array}$ & $\begin{array}{l}\text { Selon vous, comment un présentateur de } \\
\text { télévision suisse romand devrait-il prononcer } \\
\text { le mot/la phrase suivant(e) ? }\end{array}$ & $\begin{array}{l}\text { Stimulus 1 vs. stimulus 2 } \\
\text { (vs. stimulus 3) }\end{array}$ \\
\hline $\begin{array}{l}\text { Français langue } \\
\text { étrangère (FLE) }\end{array}$ & $\begin{array}{l}\text { Selon vous, laquelle des deux prononciations } \\
\text { du mot/de la phrase suivant(e) serait la } \\
\text { plus adaptée à être enseignée à des non- } \\
\text { francophones à Paris/en Suisse romande/au } \\
\text { Québec? }\end{array}$ & $\begin{array}{l}\text { Stimulus 1 vs. stimulus 2 } \\
\text { (vs. stimulus 3) }\end{array}$ \\
\hline
\end{tabular}

\subsubsection{Informations sociodémographiques}

Contrairement au cas du questionnaire écrit (cf. 3.3), les questions sociodémographiques ont été placées avant le test de perception afin de pouvoir exploiter également les données des informateurs ayant interrompu prématurément le test, de telles interruptions étant très courante dans le cas de questionnaires en ligne (cf. Diekmann ${ }^{6} 2012$, 434-488). Les données sociodémographiques suivantes ont été récoltées : sexe, âge, lieu dans lequel les informateurs ont passé la plus grande partie de leur enfance, domicile actuel et nombre d'années passées à cet endroit, niveau d'éducation, profession, origines du père et de la mère.

\subsubsection{Sélection et recrutement des informateurs}

La sélection des informateurs s'est faite selon le principe de l'échantillonnage par quotas déjà utilisé pour les questionnaires écrits (cf. 3.3.2). Ainsi, 96 informateurs ont été recrutés dans chacune des régions. Ces informateurs ont été répartis sur quatre points d'enquête par région (24 informateurs par point d'enquête), selon les critères du sexe (deux catégories : femmes vs. hommes), de l'âge (trois catégories : 20-39, 40-59, 60+) et du niveau d'éducation (deux catégories : diplôme universitaire et diplôme de fin d'études secondaires vs. diplôme inférieur ou aucun diplôme) (cf. tableau 11). Au total, les données des tests de perception de 288 informateurs ont été retenues. Le critère déterminant la prise en compte ou non des données des informateurs était que ces derniers devaient, comme dans le cas du questionnaire écrit, avoir vécu la majeure partie des dix dernières années dans 
le quartier, la commune, la ville ou l'agglomération urbaine présentée dans le chapitre 3.3.2 et avoir au minimum un parent (père et/ou mère) ayant vécu la plus grande partie de son enfance dans cette localité. Les auditeurs ne présentant pas le profil recherché ont ainsi été exclus du traitement des données, tout comme les informateurs présentant un profil déjà rempli dans les quotas.

Pour des raisons d'économie et de praticabilité, contrairement au cas du questionnaire écrit, le recrutement des informateurs a été effectué aussi bien hors ligne qu'en ligne : une majorité des informateurs a tout d'abord été sélectionnée personnellement selon la méthode de recherche aléatoire et rapide dans les universités, bibliothèques, cafés, bars et dans la rue (cf. Postlep 2010, 98-99). Par ailleurs, cette recherche a été combinée à un recrutement aléatoire et non personnel aussi bien en ligne (sur différents portails) qu'hors ligne par le biais d'établissements publics ou privés d'enseignement. Une fois plusieurs quotas remplis, la recherche s'est faite de manière ciblée afin de trouver des informateurs correspondant aux profils manquants.

Étant donnée cette méthode hybride de recrutement, la réalisation du test de perception a également été effectuée de deux manières : à l'aide d'une tablette numérique et d'un casque fermé pour les informateurs recrutés hors ligne et sur l'ordinateur personnel pour les informateurs recrutés en ligne. Mais quel que soit le type de recrutement et le support utilisé, les tests de perception en tant que tels ont été effectués en ligne sur la plateforme SoSci Survey (cf. SoSci Survey GmbH 2018). Les informateurs ont mis entre 15 et 25 minutes pour remplir le questionnaire dans son intégralité.

\subsubsection{Protocole d'analyse}

Étant donné que la récolte des données s'est faite automatiquement par le biais de la plateforme SoSci Survey (cf. SoSci Survey GmbH 2018), ces données ont pu être extraites directement dans un format .csv, retravaillées sous Excel et importées dans le programme statistique $R$. Elles ont par la suite été soumises à des analyses statistiques afin de mesurer la signification des résultats ainsi que les éventuels effets de facteurs externes (sexe, âge, origine et niveau d'éducation des informateurs) ayant influencé ces résultats. Les possibilités de réponses aux questions ont été utilisées en tant que variable dépendante et les données sociodémographiques en tant que variables explicatives indépendantes. Différents modèles statistiques ont été appliqués aux données, en fontion du type de variable dépendante (quantitative continue, qualitative binaire ou qualitative à trois modalités non ordonnées) et de variables explicatives (variables qualitatives et quantitatives) : 
- Question 1: dans le cadre de la première question, comme il s'agissait de comparer plusieurs moyennes et de tester l'influence de quatre facteurs sur ces résultats, une analyse de variance (ANOVA) à quatre facteurs a été appliquée aux données brutes. Les quatre facteurs, qui sont tous de nature catégorique, sont le sexe (femmes vs. hommes), l'âge (20-39; 40-59; 60+), l'origine (quatre localités par région) et le niveau d'éducation (deux niveaux d'éducation par région) des informateurs.

- Questions 2 et 3 : les catégories de réponses de la deuxième et troisième question sont dans la grande majorité des cas de nature qualitative binaire, étant donné que les informateurs avaient la possibilité de choisir une variante après en avoir entendu deux en opposition. Ainsi, une régression logistique binaire a été appliquée à ces données à l'aide du modèle linéaire généralisé glm avec réponse binomiale et lien logit et en prenant en compte les quatre variables sociodémographiques (sexe, âge, origine, niveau d'éducation). Dans le cas de Paris, les variantes de deux groupes sociodémographiques ont été comparées les unes avec les autres et plusieurs questions regroupent donc plus de deux stimuli. Dans ces cas-ci, le choix du modèle s'est porté sur une régression logistique multinomiale à l'aide de la fonction polr de l'extension MASS. 